\title{
Scalar, Electromagnetic and Gravitational Perturbations of Kerr-Newman Black Holes in the Slow-Rotation Limit
}

\author{
Paolo Pani, ${ }^{1,2}$, 田 Emanuele Berti, ${ }^{3,4}$, 团 and Leonardo Gualtieri 5 , 团 \\ ${ }^{1}$ CENTRA, Departamento de Física, Instituto Superior Técnico, \\ Universidade Técnica de Lisboa - UTL, Avenida Rovisco Pais 1, 1049 Lisboa, Portugal. \\ ${ }^{2}$ Institute for Theory \& Computation, Harvard-Smithsonian CfA, 60 Garden Street, Cambridge, MA, USA \\ ${ }^{3}$ Department of Physics and Astronomy, The University of Mississippi, University, MS 3867\%, USA. \\ ${ }^{4}$ California Institute of Technology, Pasadena, CA 91109, USA \\ ${ }^{5}$ Dipartimento di Fisica, Università di Roma "La Sapienza" E Sezione INFN Roma1, P.A. Moro 5, 00185, Roma, Italy.
}

(Dated: July 30, 2013)

\begin{abstract}
In Einstein-Maxwell theory, according to classic uniqueness theorems, the most general stationary black-hole solution is the axisymmetric Kerr-Newman metric, which is defined by three parameters: mass, spin and electric charge. The radial and angular dependence of gravitational and electromagnetic perturbations in the Kerr-Newman geometry do not seem to be separable. In this paper we circumvent this problem by studying scalar, electromagnetic and gravitational perturbations of Kerr-Newman black holes in the slow-rotation limit. We extend (and provide details of) the analysis presented in a recent Letter [1]. Working at linear order in the spin, we present the first detailed derivation of the axial and polar perturbation equations in the gravito-electromagnetic case, and we compute the corresponding quasinormal modes for any value of the electric charge. Our study is the first self-consistent stability analysis of the Kerr-Newman metric, and in principle it can be extended to any order in the small rotation parameter. We find numerical evidence that the axial and polar sectors are isospectral at first order in the spin, and speculate on the possible implications of this result.
\end{abstract}

PACS numbers: $04.70 . \mathrm{Bw}, 04.25 . \mathrm{Nx}, 04.30 . \mathrm{Db}$

\section{INTRODUCTION}

\section{A. Motivation and Background}

Classic uniqueness theorems (reviewed in, e.g., [2]) guarantee that the Kerr-Newman (KN) metric [3] describes the most general stationary electrovacuum solution in Einstein-Maxwell theory. The KN line element

$$
\begin{aligned}
& d s^{2}=-d t^{2}+\Sigma\left(\frac{d r^{2}}{\Delta}+d \vartheta^{2}\right)+\left(r^{2}+a^{2}\right) \sin ^{2} \vartheta d \varphi^{2} \\
& +\frac{2 M r-Q^{2}}{\Sigma}\left(d t-a \sin ^{2} \vartheta d \varphi\right)^{2},
\end{aligned}
$$

(where $\Sigma=r^{2}+a^{2} \cos ^{2} \vartheta, \Delta=r^{2}+a^{2}-2 M r+Q^{2}$ ) is characterized by 3 parameters: mass $M$, angular momentum $J=M a$ and electromagnetic charge $Q$. In the neutral case $(Q=0)$ the $\mathrm{KN}$ solution reduces to the Kerr metric, whereas in the nonspinning limit $(J=0)$ it reduces to the Reissner-Nordström (RN) metric. When both $Q \neq 0$ and $J \neq 0$ the spacetime is endowed with a magnetic dipole moment, and it has the same gyromagnetic ratio $g=2$ as the electron [4]. This has led to some speculation that the KN metric could be used as a classical model for elementary particles [5].

Most work in BH physics has focused on the Kerr metric, because astrophysical BHs are likely to be electrically

\footnotetext{
* paolo.pani@ist.utl.pt

† berti@phy.olemiss.edu

$\ddagger$ Leonardo.Gualtieri@roma1.infn.it
}

neutral. The reason is that a $\mathrm{BH}$ of mass $M$ and charge $Q$ will not gravitationally accrete particles of mass $m$ and charge $e$ as long as $e Q>M m$ (in the natural units $G=c=1$ used in this paper). Since $m / e \sim 10^{-21}$ for electrons, large BHs will hardly acquire any charge (see e.g. [6]). Furthermore, in astrophysical environments the electric charge is expected to be shorted out by the surrounding plasma [7].

Notwithstanding the fact that charge is unlikely to play a significant role in astrophysics, the KN metric is a precious theoretical laboratory to investigate the dynamics of Einstein-Maxwell theory in curved spacetime, and the linearized dynamics of test fields on a KN background have been intensively studied in the past.

The KN metric is the only nontrivial, asymptotically flat solution of the Einstein-Maxwell system for which the geodesic and Klein-Gordon equations can be solved by separation of variables [8]. The neutrino [9], massive spin-1/2 [10, 11] and Rarita-Schwinger [12] equations in the KN metric are also known to be separable. The separability of fermionic fields is related to the existence of a generalized total angular momentum operator for the Dirac equation in curved spacetime, that satisfies appropriate conservation laws [13, 14]. Scalar and Dirac perturbations of a KN BH can therefore be treated using the same general methods that apply to Kerr BHs. In particular, it is straightforward to compute the quasinormal modes (QNMs) of KN BHs for these classes of perturbations (see [15 19] for reviews on QNMs). For the KN metric, scalar QNMs were computed in [20] (see also Ref. 21] for a recent generalization to massive and charged scalar 
QNMs) and Dirac QNMs were computed in [22]. The superradiant instability of massive scalar fields was studied in 23]. Hartman et al. studied the scattering of charged scalars and fermions in near-extremal KN spacetimes 24], complementing earlier work on the so-called Kerr-Newman/Conformal Field Theory (KN/CFT) conjecture [25]. Because the equations are separable, (the absence of) superradiant effects [26] and greybody factors for charged and massive Dirac particles in KN have also been studied extensively [5, 27 [36]: see [37] for a good overview of work in this field, and [38] for a study of Dirac perturbations of KN BHs in anti-de Sitter space.

Much less is known about the gravitationalelectromagnetic sector of KN perturbations. The reason is that most methods to compute QNMs, greybody factors and scattering amplitudes (including the continued fraction method 39] and the asymptotic iteration method [40]) rely on the separability of the perturbation equations. Despite several attempts [4] 43 ], no one has succeeded at separating the angular and radial dependence of the gravitational-electromagnetic eigenfunctions. Chandrasekhar's monograph [44] gives a comprehensive overview of this long-standing unsolved problem.

A relatively small number of papers tried to address the problem of the oscillations and stability of the KN metric under the combined effect of electromagnetic and gravitational perturbations. Mashhoon [45] used the analogy between geodesic stability and QNMs first proposed by Goebel [46] (see also [47]) to estimate the QNMs in the eikonal approximation and to argue that the KN metric should be stable. Dudley and Finley (41, 42], henceforth DF) made a remarkable study of the separability of linear perturbations of the solutions of the Einstein-Maxwell equations found by Plebánski and Demiánski [48], which include all vacuum Type D solutions in the Petrov classification. Due to nonseparability of the perturbation equations, they could not treat gravito-electromagnetic perturbations in a fully consistent approach. In their work, DF "either keep the geometry fixed and perturb the electric field or, of more interest, keep the electric field fixed and perturb the geometry". This approach should be appropriate for values of the charge $Q$ at most as large as the perturbations of the spacetime metric. Kokkotas first used the DF equation to compute the fundamental gravitational QNM using WKB methods [49]. Later on, Berti and Kokkotas [20] confirmed that the WKB approximation is reasonably accurate for all values of the dimensionless spin parameter $\left(\tilde{a} \equiv J / M^{2}\right)$ and of the charge $Q$ by comparing WKB results to a continued-fraction solution. The main problem of the DF approach is not computational, but physical. The DF equation does not treat the gravito-electromagnetic coupling in a self-consistent way (for example, it does not reduce to the well-known RN perturbation equations as $J \rightarrow 0$ ). Therefore it is unclear whether it provides a correct description of gravitational and electromagnetic perturbations of KN BHs.

In a recent Letter [1], we have presented the first self- consistent study of the gravito-electromagnetic perturbations of KN BHs. This paper complements and extends the results of [1], providing details of the derivation of the perturbation equations and a more comprehensive set of numerical results.

Our approach relies on a clear physical approximation, i.e. a slow-rotation expansion of the perturbations of spinning BHs [50, 51]. The formalism to address this problem was originally proposed in the context of slowly rotating compact stars 52 55], and it can be extended (at least in principle) to any perturbative order in the small rotation parameter. Within the slow-rotation expansion (which is valid for any value of the $\mathrm{BH}$ charge $Q$ ) it is possible to estimate truncation errors, e.g. by extending the computation to the next order in rotation or by comparison with cases where a nonperturbative solution is available (such as the case of scalar perturbations considered below).

\section{B. Executive Summary}

In the remainder of this Introduction we provide a short executive summary of our main results, that is also meant as a guide to the structure of the paper.

In Section [I] we review our approach to separate the scalar and the gravito-electromagnetic perturbations of a $\mathrm{KN} \mathrm{BH}$ in the slow-rotation limit [50, 51, 53]. We derive the equations describing gravito-electromagnetic oscillations in the slow-rotation approximation by linearizing the Einstein-Maxwell equations with respect to both the amplitude of the oscillation and the $\mathrm{BH}$ spin parameter $\tilde{a} \equiv J / M^{2}$. By expanding the perturbations of the spacetime metric and of the electromagnetic field in tensor spherical harmonics, we obtain a coupled system of differential equations. Our main analytical result is the derivation of two sets of coupled, second-order equations (one for the axial and one for the polar sector, respectively) which fully describe gravito-electromagnetic perturbations of a $\mathrm{KN} \mathrm{BH}$ to first order in the spin:

$$
\begin{aligned}
\hat{\mathcal{D}} Z_{i}^{ \pm} & \equiv V_{0}^{(i, \pm)} Z_{i}^{ \pm}+m \tilde{a}\left[V_{1}^{(i, \pm)} Z_{i}^{ \pm}+V_{2}^{(i, \pm)} Z_{i}^{ \pm^{\prime}}\right] \\
& +m \tilde{a} Q^{2}\left[W_{1}^{(i, \pm)} Z_{j}^{ \pm}+W_{2}^{(i, \pm)} Z_{j}^{ \pm^{\prime}}\right]
\end{aligned}
$$

Here $i, j=1,2, i \neq j$ and there is no sum over the indices $i, j$. A prime denotes a derivative with respect to $r$, and we have introduced the differential operator

$$
\hat{\mathcal{D}}=\frac{d^{2}}{d r_{*}^{2}}+\omega^{2}-F \frac{\ell(\ell+1)}{r^{2}} .
$$

where $r_{*}$ is the standard tortoise coordinate defined as $d r / d r_{*}=\left(r-r_{-}\right)\left(r-r_{+}\right) / r^{2}$, and $r_{ \pm}=M \pm \sqrt{M^{2}-Q^{2}}$ are the outer $\left(r_{+}\right)$and Cauchy $\left(r_{-}\right)$horizons of a $\mathrm{RN}$ $\mathrm{BH}$. The functions $Z_{i}^{-}$and $Z_{i}^{+}$are linear combinations of axial and polar variables, respectively, and they are also combinations of gravitational and electromagnetic 

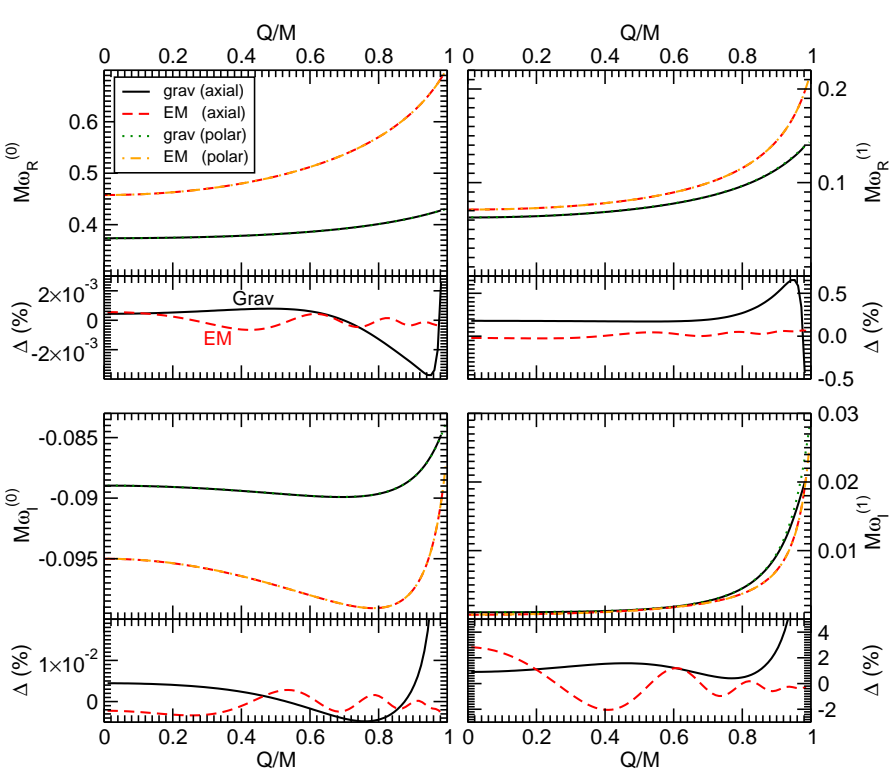
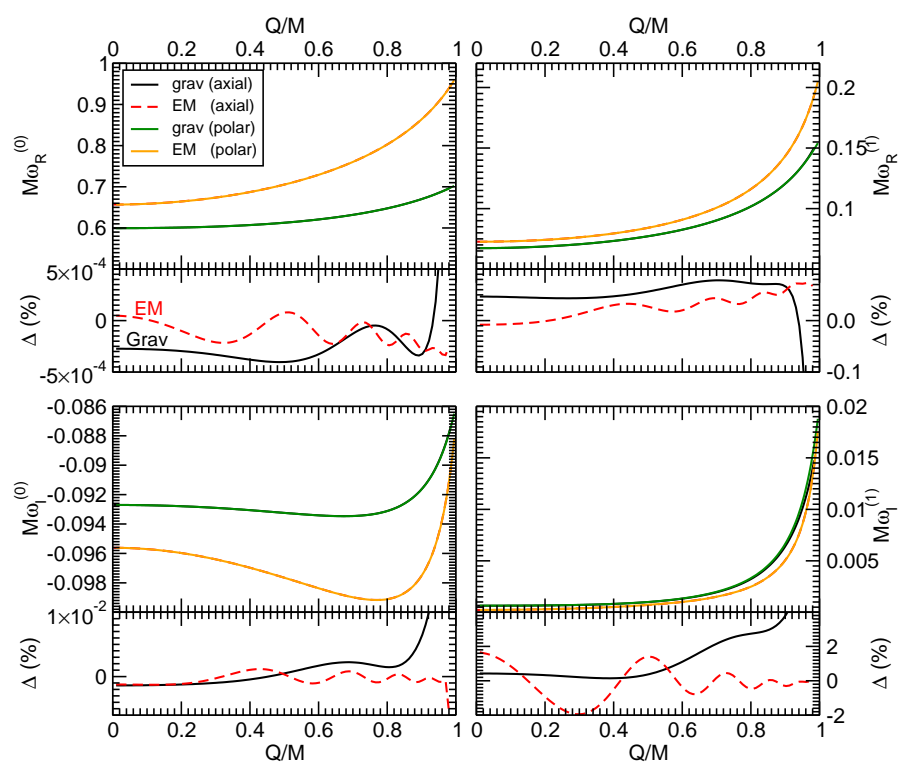

FIG. 1. Left (reproduced from Ref. [1]): Zeroth-order (small left panels) and first-order (small right panels) terms of the slow-rotation expansion of the KN QNM frequencies [cf. Eqs. (4) and (5)]. All quantities are plotted as a function of $Q / M$, and they refer to the fundamental mode $(n=0)$ with $\ell=2$. The lower part of each panel shows the percentage difference between axial and polar quantities: our results are consistent with isospectrality to $\mathcal{O}(0.1 \%)$ for the real part and to $\mathcal{O}(1 \%)$ for the imaginary part of these modes. Right: same, but for fundamental modes with $\ell=3$.

perturbations. A step-by-step derivation of these equations is presented in Appendix $\mathrm{A}$ and in a publicly available Mathematica notebook [56]. The axial potentials $V^{(i,-)}$ and $W^{(i,-)}$ are listed in Appendix A1, while the polar potentials $V^{(i,+)}$ and $W^{(i,+)}$ are so lengthy that we decided to make them available only through the MATHEMATICA notebook [56] in order to save space.

We have integrated the coupled system (2) and computed the corresponding eigenfrequencies using two independent methods, which are described in Section III (see also Ref. [57] for a review). For any value of $Q$, our analysis allows us to extract the first-order corrections to the complex QNM frequencies $\omega=\omega_{R}+i \omega_{I}$ :

$$
\begin{gathered}
\omega_{R}=\omega_{R}^{(0)}+\tilde{a} m \omega_{R}^{(1)}+\mathcal{O}\left(\tilde{a}^{2}\right), \\
\omega_{I}=\omega_{I}^{(0)}+\tilde{a} m \omega_{I}^{(1)}+\mathcal{O}\left(\tilde{a}^{2}\right),
\end{gathered}
$$

where $\omega_{R}^{(i)}$ and $\omega_{I}^{(i)}$ are functions of $Q$ and of the multipolar index $\ell$, and the $m$-dependence has been factored out.

Section IV presents our numerical results. We begin by studying scalar perturbations of $\mathrm{KN} \mathrm{BHs}$, for which the perturbation equations are separable and QNM frequencies can be computed "exactly" in the Teukolsky formalism [20]. By comparing QNM frequencies in the slow-rotation approximation to the Teukolsky-based results, we find that the relative error of the slow-rotation approximation is less than $1 \%$ as long as $J / J_{\max } \lesssim 0.3$, where $J_{\max }$ is the maximum allowed $\mathrm{KN}$ spin for any fixed value of the electric charge.

Figure 1 shows our main numerical results for the fundamental gravito-electromagnetic perturbations with $\ell=2,3$, which are the most relevant for gravitationalwave emission (see e.g. [58]). In each panel we show four curves, corresponding to the axial and polar "gravitational" and "electromagnetic" modes (as defined in the decoupled $Q=0$ limit: see Section IV for details). The zeroth-order terms shown in the small left panels are simply RN QNMs; they agree with continued-fraction solutions of the equations first derived by Zerilli [59], as computed by Leaver [60].

Gravito-electromagnetic perturbations of nonspinning $\mathrm{BHs}$ in general relativity have a noteworthy property that was proved by Chandrasekhar [44]: the polar and axial potentials can be written in terms of a superpotential, which implies that the polar and axial QNMs are isospectral [18]. A priori, there is no reason why such a remarkable property should hold true also for KN BHs.

In addition to computing the QNMs of a $\mathrm{KN} B H$ in a full consistent setting for the first time, perhaps the most important result of our numerical study is strong evidence that the axial and polar sectors of $\mathrm{KN}$ gravitoelectromagnetic perturbations are indeed isospectral to first order in the BH spin. In the inset of Fig. 1 we show the relative difference between the coefficients of axial and polar modes as functions of $Q$ : our results are consistent with isospectrality within the numerical errors implicit in our method. This is further discussed in Section IV] where we provide additional evidence that higher multipoles (with $\ell>2$ ) and higher overtones (for a given $\ell)$ are also isospectral to first order in rotation.

These numerical results lead us to the tantalizing con- 
jecture that the modes of a $\mathrm{KN} \mathrm{BH}$ may be isospectral to any order in $\tilde{a}$. We conclude our paper in Section V with a brief discussion of this conjecture and of possible ways to put it to the test (see also [1] ).

\section{FORMALISM}

Let us consider Einstein-Maxwell theory:

$$
S=\int d^{4} x \sqrt{-g}\left(R-F_{\mu \nu} F^{\mu \nu}\right),
$$

where $R$ is the Ricci scalar and $F_{\mu \nu}=\partial_{\mu} A_{\nu}-\partial_{\nu} A_{\mu}$ is the Maxwell field strength. The KN metric (1) is the most general stationary electrovacuum solution of this theory. Here and in the following we linearize in the spin parameter $\tilde{a} \equiv J / M^{2}$, neglecting terms of order $\mathcal{O}\left(\tilde{a}^{2}\right)$. To this order, the KN metric reads

$$
\begin{aligned}
d s_{0}^{2}=g_{\mu \nu}^{(0)} d x^{\mu} d x^{\nu}= & -F(r) d t^{2}+F(r)^{-1} d r^{2}+r^{2} d^{2} \Omega \\
& -2 \varpi(r) \sin ^{2} \vartheta d \varphi d t
\end{aligned}
$$

where

$$
\begin{aligned}
& F(r)=1-\frac{2 M}{r}+\frac{Q^{2}}{r^{2}}, \\
& \varpi(r)=\frac{2 \tilde{a} M^{2}}{r}-\frac{\tilde{a} Q^{2} M}{r^{2}},
\end{aligned}
$$

and the background electromagnetic potential is given by

$$
A_{\mu}=\left(\frac{Q}{r}, 0,0,-\frac{\tilde{a} Q M}{r} \sin ^{2} \vartheta\right) .
$$

The magnetic field is given by the curl of the threepotential above. Thus, the presence of both rotation and charge $(\tilde{a} Q \neq 0)$ induces a magnetic field in the $(\vartheta, \varphi)$ directions.

\section{A. Harmonic decomposition}

We linearize the metric as

$$
g_{\mu \nu}=g_{\mu \nu}^{(0)}+h_{\mu \nu}
$$

and we decompose the metric perturbations $h_{\mu \nu}$ in the Regge-Wheeler gauge:

$$
h_{\mu \nu}=\left(\begin{array}{cc|cc}
H_{0}^{\ell} Y^{\ell} & H_{1}^{\ell} Y^{\ell} & h_{0}^{\ell} S_{\vartheta}^{\ell} & h_{0}^{\ell} S_{\varphi}^{\ell} \\
H_{1}^{\ell} Y^{\ell} & H_{2}^{\ell} Y^{\ell} & h_{1}^{\ell} S_{\vartheta}^{\ell} & h_{1}^{\ell} S_{\varphi}^{\ell} \\
\hline h_{0}^{\ell} S_{\vartheta}^{\ell} & h_{1}^{\ell} S_{\vartheta}^{\ell} & r^{2} K^{\ell} Y^{\ell} & 0 \\
h_{0}^{\ell} S_{\varphi}^{\ell} & h_{1}^{\ell} S_{\varphi}^{\ell} & 0 & r^{2} K^{\ell} \sin ^{2} \vartheta Y^{\ell}
\end{array}\right),
$$

where $Y^{\ell}=Y^{\ell}(\vartheta, \varphi)$ are the ordinary scalar spherical harmonics, $\left(S_{\vartheta}^{\ell}, S_{\varphi}^{\ell}\right) \equiv\left(-Y_{, \varphi}^{\ell} / \sin \vartheta, \sin \vartheta Y_{, \vartheta}^{\ell}\right)$ are the axial vector harmonics, and $H_{0,1,2}^{\ell}, h_{0,1}^{\ell}, K^{\ell}$ are functions of $(t, r)$. Here and in the following, a sum over the harmonic indices $\ell$ and $m$ (with $|m|<\ell$ ) is implicit. We will append the relevant multipolar index $\ell$ to any perturbation variable but omit the index $m$, because in an axisymmetric background it is possible to decouple the perturbation equations so that all quantities have the same value of $m$. 61]:

We expand the electromagnetic potential as follows

$$
\delta A_{\mu}(t, r, \vartheta, \varphi)=\left[\begin{array}{c}
0 \\
0 \\
u_{(4)}^{\ell} S_{b}^{\ell} / \Lambda
\end{array}\right]+\left[\begin{array}{c}
u_{(1)}^{\ell} Y^{\ell} / r \\
u_{(2)}^{\ell} Y^{\ell} /(r F) \\
u_{(3)}^{\ell} Y_{b}^{\ell} / \Lambda
\end{array}\right]
$$

where $\Lambda=\ell(\ell+1), b=(\vartheta, \varphi), Y_{b}^{\ell}=\left(Y_{, \vartheta}^{\ell}, Y_{, \varphi}^{\ell}\right)$ are the polar vector harmonics, and $u_{(1,2,3,4)}^{\ell}$ are functions of $(t, r)$. Inserting the harmonic expansion of the metric perturbations (12) and of the Maxwell field (13) into the linearized Einstein equations we find the equations for the perturbation functions to linear order in $\tilde{a}$. The latter naturally separate into three groups [50, 51, 53, 57]. By denoting the linearized Einstein equations as $\delta \mathcal{E}_{\mu \nu}=0$, the first group is formed by the equations $\delta \mathcal{E}_{(I)}=0$, where $I=0,1,2,3$ is shorthand notation for $(t t),(t r),(r r)$ and $(+)$, respectively, and we have defined $\delta \mathcal{E}_{(+)} \equiv$ $\delta \mathcal{E}_{\vartheta \vartheta}+\delta \mathcal{E}_{\varphi \varphi} / \sin ^{2} \vartheta$. The equations can be cast in the form

$\delta \mathcal{E}_{(I)} \equiv\left(A_{\ell}^{(I)}+\tilde{A}_{\ell}^{(I)} \cos \vartheta\right) Y^{\ell}+B_{\ell}^{(I)} \sin \vartheta Y_{, \vartheta}^{\ell}+C_{\ell}^{(I)} Y_{, \varphi}^{\ell}=0$

where $A_{\ell}^{(I)}, \tilde{A}_{\ell}^{(I)}, B_{\ell}^{(I)}, C_{\ell}^{(I)}$ are combinations of the perturbation functions $\left\{H_{0,1,2}^{\ell}, h_{0,1}^{\ell}, K^{\ell}, u_{(1,2,3,4)}^{\ell}\right\}$. The second group is formed by the equations $\delta \mathcal{E}_{L \vartheta}=0$, with $L=0,1$ corresponding to the $t, r$ components, respectively. They can be cast in the form

$$
\begin{aligned}
\delta \mathcal{E}_{(L \vartheta)} & \equiv\left(\alpha_{\ell}^{(L)}+\tilde{\alpha}_{\ell}^{(L)} \cos \vartheta\right) Y_{, \vartheta}^{\ell}-\left(\beta_{\ell}^{(L)}+\tilde{\beta}_{\ell}^{(L)} \cos \vartheta\right) \frac{Y_{, \varphi}^{\ell}}{\sin \vartheta} \\
& +\eta_{\ell}^{(L)} \sin \vartheta Y^{\ell}+\xi_{\ell}^{(L)} X^{\ell}+\chi_{\ell}^{(L)} \sin \vartheta W^{\ell}=0, \\
\delta \mathcal{E}_{(L \varphi)} & \equiv\left(\beta_{\ell}^{(L)}+\tilde{\beta}_{\ell}^{(L)} \cos \vartheta\right) Y_{, \vartheta}^{\ell}+\left(\alpha_{\ell}^{(L)}+\tilde{\alpha}_{\ell}^{(L)} \cos \vartheta\right) \frac{Y_{, \varphi}^{\ell}}{\sin \vartheta} \\
& +\zeta_{\ell}^{(L)} \sin \vartheta Y^{\ell}+\chi_{\ell}^{(L)} X^{\ell}-\xi_{\ell}^{(L)} \sin \vartheta W^{\ell}=0,
\end{aligned}
$$

where $\alpha_{\ell}^{(L)}, \tilde{\alpha}_{\ell}^{(L)}, \beta_{\ell}^{(L)}, \tilde{\beta}_{\ell}^{(L)}, \eta_{\ell}^{(L)}, \xi_{\ell}^{(L)}, \zeta_{\ell}^{(L)}, \chi_{\ell}^{(L)}$ are combinations of the perturbation functions. The third group is (defining $\delta \mathcal{E}_{(-)} \equiv \delta \mathcal{E}_{\vartheta \vartheta}-\delta \mathcal{E}_{\varphi \varphi} / \sin ^{2} \vartheta$ )

$$
\begin{aligned}
\delta \mathcal{E}_{(\vartheta \varphi)} & \equiv f_{\ell} \sin \vartheta \partial_{\vartheta} Y^{\ell}+g_{\ell} Y_{, \varphi}^{\ell}+s_{\ell} \frac{X^{\ell}}{\sin \vartheta}+t_{\ell} W^{\ell}=0 \\
\delta \mathcal{E}_{(-)} & \equiv g_{\ell} \sin \vartheta \partial_{\vartheta} Y^{\ell}-f_{\ell} Y_{, \varphi}^{\ell}-t_{\ell} \frac{X^{\ell}}{\sin \vartheta}+s_{\ell} W^{\ell}=0
\end{aligned}
$$


where we have defined

$$
\begin{aligned}
X^{\ell} & \equiv 2\left(Y_{, \vartheta \varphi}^{\ell}-\cot \vartheta Y_{, \varphi}^{\ell}\right), \\
W^{\ell} & \equiv Y_{, \vartheta \vartheta}^{\ell}-\cot \vartheta Y_{, \vartheta}^{\ell}-\frac{Y_{, \varphi \varphi}^{\ell}}{\sin ^{2} \vartheta} .
\end{aligned}
$$

The linearized Maxwell equations can be also recast in a similar form. The $t$ - and $r$-components belong to the first group and can be arranged in the form of Eq. (14) with $I=4,5$, respectively. The $\vartheta$ - and $\varphi$ - components belong to the second group, and can be written as in Eq. (15) and (16) with $L=2$. The coefficients can be split into two sets:

$\begin{array}{cllll}\text { Polar: } & A_{\ell}^{(I)}, & C_{\ell}^{(I)}, & \alpha_{\ell}^{(L)}, & \tilde{\beta}_{\ell}^{(L)}, \\ & \zeta_{\ell}^{(L)}, & \xi_{\ell}^{(L)}, & s_{\ell}, \quad f_{\ell}, \\ \text { Axial: } & \tilde{A}_{\ell}^{(I)}, & B_{\ell}^{(I)}, & \beta_{\ell}^{(L)}, & \tilde{\alpha}_{\ell}^{(L)}, \\ & \eta_{\ell}^{(L)}, & \chi_{\ell}^{(L)}, & t_{\ell}, & g_{\ell},\end{array}$

where henceforth the indices $I=0, \ldots, 5$ and $L=0,1,2$ account for both the Einstein and Maxwell equations. The explicit form of the coefficients is not particularly illuminating, and it is given in a publicly available МATHEMATICA notebook [56]. The crucial point is to recognize that the coefficients above are purely radial functions, i.e. the entire angular dependence has been factored out from the field equations.

\section{B. Separation of the angular dependence}

The separation of the angular dependence of Einstein's equations for a slowly-rotating star was performed in Ref. [53]. The procedure has been extended to general slowly rotating BH solutions in Refs. [50, 51] (see also [57]); we refer the reader to those papers for details. Using the orthogonality properties of scalar spherical harmonics, from Eq. (14) we get

$$
\begin{aligned}
& A_{\ell}^{(I)}+i m C_{\ell}^{(I)}+\mathcal{Q}_{\ell}\left[\tilde{A}_{\ell-1}^{(I)}+(\ell-1) B_{\ell-1}^{(I)}\right]+ \\
& \mathcal{Q}_{\ell+1}\left[\tilde{A}_{\ell+1}^{(I)}-(\ell+2) B_{\ell+1}^{(I)}\right]=0
\end{aligned}
$$

where we have defined $\mathcal{Q}_{\ell}^{2}=\left(\ell^{2}-m^{2}\right) /\left(4 \ell^{2}-1\right)$. From the orthogonality of vector spherical harmonics and using
Eqs. (15)-(16) we get

$$
\begin{aligned}
& \Lambda \alpha_{\ell}^{(L)}+i m\left[(\ell-1)(\ell+2) \xi_{\ell}^{(L)}-\tilde{\beta}_{\ell}^{(L)}-\zeta_{\ell}^{(L)}\right]+ \\
& \mathcal{Q}_{\ell}(\ell+1)\left[(\ell-2)(\ell-1) \chi_{\ell-1}^{(L)}+(\ell-1) \tilde{\alpha}_{\ell-1}^{(L)}-\eta_{\ell-1}^{(L)}\right]- \\
& \mathcal{Q}_{\ell+1} \ell\left[(\ell+2)(\ell+3) \chi_{\ell+1}^{(L)}-(\ell+2) \tilde{\alpha}_{\ell+1}^{(L)}-\eta_{\ell+1}^{(L)}\right]=0, \\
& \Lambda \beta_{\ell}^{(L)}+i m\left[(\ell-1)(\ell+2) \chi_{\ell}^{(L)}+\tilde{\alpha}_{\ell}^{(L)}+\eta_{\ell}^{(L)}\right]- \\
& \mathcal{Q}_{\ell}(\ell+1)\left[(\ell-2)(\ell-1) \xi_{\ell-1}^{(L)}-(\ell-1) \tilde{\beta}_{\ell-1}^{(L)}+\zeta_{\ell-1}^{(L)}\right]+ \\
& \mathcal{Q}_{\ell+1} \ell\left[(\ell+2)(\ell+3) \xi_{\ell+1}^{(L)}+(\ell+2) \tilde{\beta}_{\ell+1}^{(L)}+\zeta_{\ell+1}^{(L)}\right]=0 .
\end{aligned}
$$

Finally, the orthogonality of tensor spherical harmonics applied to Eqs. (17) and (18) yields

$$
\begin{aligned}
& 0=\Lambda s_{\ell}-i m f_{\ell}-\mathcal{Q}_{\ell}(\ell+1) g_{\ell-1}+\mathcal{Q}_{\ell+1} \ell g_{\ell+1}, \\
& 0=\Lambda t_{\ell}+i m g_{\ell}-\mathcal{Q}_{\ell}(\ell+1) f_{\ell-1}+\mathcal{Q}_{\ell+1} \ell f_{\ell+1} .
\end{aligned}
$$

Summarizing, once we fix the value of $m$, truncating the expansion in $\ell$ to a value $\ell_{\max }$, the index $\ell$ can have $n_{\ell}=$ $\ell_{\max }-|m|+1$ possible values; our separation procedure in the slow-rotation limit yields a system of $14 n_{\ell}$ coupled, ordinary differential equations (10 for the gravitational sector and 4 for the Maxwell sector for each $\ell$ ), given in Eqs. (21), (22)-(23) and (24)-(25). Their explicit form is available online [56].

\section{First-order corrections to the eigenvalue equations}

In the case of slow rotation $(\tilde{a} \ll 1)$, a Laporte-like selection rule couples perturbations with harmonic index $\ell$ with those having opposite parity and harmonic index $\ell \pm 1$ [50 53]. This is a consequence of using a basis of spherical harmonics in a nonspherical background. However, as discussed in detail in [51, 54, 62], the couplings to the $\ell \pm 1$ terms do not contribute to the QNM spectrum to first order in $\tilde{a}$. For this reason, we shall neglect these terms in the following. In this way we get a system of 14 coupled differential equations for any chosen value of $\ell$.

Once the couplings among different $\ell$ 's are neglected, we can simply fix a value of $\ell$ (and in order to simplify the notation we will drop the index $\ell$ from all perturbation functions). We perform a Fourier decomposition by assuming that all perturbations have a time dependence $\sim e^{-i \omega t}$.

As we explicitly show in Appendix $\mathrm{A}$ axial and polar perturbations decouple, and Eqs. (21)-(25) can be reduced to two coupled, second-order differential equations (one pair for each parity), that we have already written down in the Introduction [Eq. (2)]. These equations display the same symmetries as the master equations for a $\mathrm{RN} \mathrm{BH}$ [44], and indeed they exactly reduce to those 
equations in the nonrotating case. We remark in passing that the DF equations previously used to investigate gravito-electromagnetic perturbations of the $\mathrm{KN}$ metric do not satisfy this requirement. Eqs. (2) are the main result of this paper. They contain two first-order corrections in the rotational parameter $\tilde{a}$. The first term couples the functions $Z_{i}^{ \pm}$with the same $Z_{i}^{ \pm}$. It is responsible for a Zeeman-like splitting of the eigenfrequencies, which breaks the degeneracy in the azimuthal index $m$. The second line in Eqs. (2) is more interesting. First, it is proportional to the combination $\tilde{a} Q^{2}$, so it is vanishing when the $\mathrm{BH}$ is nonspinning or uncharged. Furthermore, this term couples the function $Z_{1}^{+}$with the function $Z_{2}^{+}$, and the function $Z_{1}^{-}$with the function $Z_{2}^{-}$.

We remark that this coupling disappears when $\tilde{a}=0$, but this does not mean that gravitational and electromagnetic perturbations decouple in that limit: whenever $Q \neq 0$, the functions $Z_{j}^{ \pm}$are combinations of electromagnetic and gravitational perturbations, which are then coupled. Electromagnetic and gravitational perturbations only decouple when $Q=0$ (e.g., for Schwarzschild or Kerr BHs) because the functions $Z_{1}^{ \pm}$describe pure gravitational perturbations, the functions $Z_{2}^{ \pm}$describe pure electromagnetic perturbations, and the coupling term in (2) vanishes (see e.g. Ref. [44]).

Despite the complicated form of Eqs. (2), their asymptotic behavior is simple. The asymptotic solutions at the horizon and at infinity are plane waves, namely:

$$
Z_{j}^{ \pm}(r) \sim\left\{\begin{array}{ll}
e^{i \omega r_{*}}, & r \rightarrow \infty \\
e^{-i\left(\omega-m \Omega_{H}\right) r_{*}}, & r \rightarrow r_{+}
\end{array} .\right.
$$

As shown by the behavior above, near the $\mathrm{BH}$ horizon we obtain the typical frame-dragging effect: a static observer at infinity would see a wave whose wave number is

$$
k_{H}=\omega-m \Omega_{H} \sim \omega-\frac{\tilde{a} m}{M\left(1+\tilde{a}_{\max }\right)^{2}}+\mathcal{O}\left(\tilde{a}^{3}\right),
$$

where we have assumed $\omega M \ll \tilde{a}$, and

$$
\tilde{a}_{\max } \equiv J_{\max } / M^{2}=\sqrt{1-(Q / M)^{2}}
$$

is the maximum spin parameter of a $\mathrm{KN} \mathrm{BH}$ and $\Omega_{H}=$ $-\lim _{r \rightarrow r_{+}} g_{t \varphi} / g_{\varphi \varphi}$ is the angular velocity at the horizon of locally nonrotating observers to first order in $\tilde{a}$. In the small-charge limit, $k_{H} \sim \omega-\tilde{a} m /(4 M)-\tilde{a} m Q^{2} /\left(8 M^{3}\right)+$ $\mathcal{O}\left[(Q / M)^{4}\right]$.

According to Eq. (26), if $\omega<m \Omega_{H}$ an observer at infinity would see waves coming out of the horizon. This corresponds to extraction of energy from a spinning $\mathrm{BH}$, resulting in superradiant amplification of the wave [26]. Therefore it is not necessary to solve the linearized field equations in order to show that superradiance in a $\mathrm{KN}$ spacetime occurs also for gravito-electromagnetic perturbations, similarly to the scalar case. A note of caution is necessary in this regard: as discussed in Ref. [51], we must include second-order terms in the expansion for a consistent treatment of superradiance in the slowrotation approximation. The reason is that the superradiance condition implies $\omega<\Omega_{H} \sim \mathcal{O}(\tilde{a})$, therefore the $\omega^{2}$ term in the wave equation becomes of the same order as $\mathcal{O}\left(\tilde{a}^{2}\right)$ terms. However the results of Ref. [51] show that (at least in some specific cases) first- and secondorder results are in qualitative (and sometimes in remarkably good quantitative) agreement even in the superradiant regime.

\section{QUASINORMAL MODE CALCULATION FOR COUPLED SYSTEMS}

After imposing physically-motivated boundary conditions at the horizon and at infinity, Eqs. (2) form an eigenvalue problem for the frequency $\omega$. Robust numerical methods to solve this class of coupled eigenvalue problems have recently been extended to $\mathrm{BH}$ spacetimes (cf. [57] for a review).

We have solved the field equations (2) by two independent techniques: a matrix-valued continued-fraction method and direct integration [51, 61].

\section{A. Matrix-valued continued fractions}

In order to reduce Eqs. (2) to a matrix-valued recurrence relation, we use the ansatz:

$$
\begin{aligned}
& Z_{j}^{ \pm}(r)= \frac{r_{+}\left(r_{+}-r_{-}\right)^{-4 i M \omega-1}}{r} e^{-2 i \omega r_{+}}\left(r-r_{-}\right)^{1+2 i M \omega} \\
& e^{i \omega r} z^{\frac{-i k_{H} r_{+}^{2}}{r_{+}-r_{-}}} \sum_{n} a_{n}^{ \pm, j} z^{n},
\end{aligned}
$$

where $z=\left(r-r_{+}\right) /\left(r-r_{-}\right)$and the \pm superscripts (not to be confused with the subscripts of the inner and outer horizon, $r_{ \pm}$) refer to the polar case ["plus" superscript in Eqs. (2)] and to the axial case ["minus" superscript in Eqs. (2)], respectively. In the axial case we obtain a tenterm matrix-valued recurrence relation, whereas in the polar case we obtain a twelve-term matrix-valued recurrence relation. They can be reduced to three-term recurrence relations using a matrix analog of Gaussian elimination [57]. Finally, the problem of finding the QNMs of the system is reduced to finding the complex roots of a determinant obtained from a nested series of inverted matrices [57].

\section{B. Direct integration}

In alternative, a direct integration technique can be shown to work very well, as long as the modes' real part is sufficiently larger than the imaginary part. This condition is typically satisfied by the fundamental mode and (to a lesser extent) by the first overtone. In this direct 
integration approach, the system (2) is integrated numerically from the horizon out to infinity. At the horizon we set the solution equal to some high-order series expansion of the form

$$
Z_{j}^{ \pm} \sim e^{-i k_{H} r_{*}} \sum_{n} b_{n}^{ \pm, j}\left(r-r_{+}\right)^{n} \quad r \rightarrow r_{+},
$$

where the coefficients $b_{n}^{ \pm, j}(n>0, j=1,2)$ can be computed in terms of $b_{0}^{ \pm, i}$ by solving the near-horizon equations order-by-order. Two independent solutions are obtained by choosing the orthonormal bases $\left(b_{0}^{ \pm, 1}, b_{0}^{ \pm, 2}\right)=$ $(1,0)$ and $\left(b_{0}^{ \pm, 1}, b_{0}^{ \pm, 2}\right)=(0,1)$. At infinity the generic behavior of the solutions reads

$Z_{j}^{ \pm} \sim B_{j}^{ \pm} e^{i \omega r_{*}} \sum_{n} \frac{c_{n}^{ \pm, j}}{r^{n}}+C_{j}^{ \pm} e^{-i \omega r_{*}} \sum_{n} \frac{d_{n}^{ \pm, j}}{r^{n}} \quad r \rightarrow \infty$.

The QNM boundary conditions correspond to imposing $C_{j}^{ \pm}=0$, i.e., purely outgoing waves at infinity. The eigenfrequencies are computed as the complex roots of (see e.g. [55])

$$
\operatorname{det}\left(\mathbf{S}^{ \pm}\right) \equiv \operatorname{det}\left(\begin{array}{ll}
C_{1}^{ \pm, 1} & C_{1}^{ \pm, 2} \\
C_{2}^{ \pm, 1} & C_{2}^{ \pm, 2}
\end{array}\right)
$$

where the superscripts denote a particular vector of the basis, i.e. $C_{j}^{ \pm, 1}$ are related to $\left(b_{0}^{ \pm, 1}, b_{0}^{ \pm, 2}\right)=(1,0)$ and $C_{j}^{ \pm, 2}$ are related to $\left(a_{0}^{ \pm, 1}, a_{0}^{ \pm, 2}\right)=(0,1)$. For any given frequency we integrate the system (2) twice and construct the matrix $\mathbf{S}^{ \pm}$. The QNMs are then obtained by imposing $\operatorname{det}\left(\mathbf{S}^{ \pm}\right)=0$, which can be achieved via a shooting method [57].

\section{NUMERICAL RESULTS}

\section{A. Scalar Quasinormal Modes in the Slow-Rotation Limit}

As a test of the slow-rotation approximation, we have computed the scalar QNMs of a $\mathrm{KN} \mathrm{BH}$ to first order in $\tilde{a}$. Since these modes can be computed exactly in the Teukolsky formalism (e.g. via continued fractions [20]), we can use these "exact" results to estimate the errors introduced by the slow-rotation approximation. For any stationary and axisymmetric spacetime, the scalar modes at first order in the angular momentum are governed by a master equation [51]. In the special case of a background given by Eq. (7), the master equation reduces to

$$
\frac{d^{2} \psi}{d r_{*}^{2}}+\left[\omega^{2}-\frac{2 m \omega \varpi(r)}{r^{2}}-F\left(\frac{\ell(\ell+1)}{r^{2}}+\frac{F^{\prime}}{r}\right)\right] \psi=0 .
$$

The corresponding eigenvalue problem can be solved with standard continued-fraction techniques [20, 60, 63]. Using the ansatz (29) for $\psi$ yields a five-term recurrence relation, whose coefficients are listed in Appendix B. The five-term recurrence relation can be reduced to a threeterm relation via standard Gaussian elimination.

In Ref. 1] we computed the relative error of the slowrotation approximation with respect to the exact result. We found a near-universal behavior of the percentage errors as functions of $J / J_{\max }=\tilde{a} / \tilde{a}_{\max }$, where $\tilde{a}_{\max }$ is the maximum allowed value of the spin parameter [corresponding to an extremal $\mathrm{KN} \mathrm{BH}$ with the given charge $Q$, cf. Eq. (28)] for all values of $Q$. This nearuniversality suggests that the parameter $\tilde{a}_{\max }[$ which appears explicitly in the QNM boundary conditions (27)] plays a fundamental role in our perturbative scheme (see also the discussion in Section IV B). In other words, the slow-rotation approximation is accurate only far from extremality, i.e. when

$$
\tilde{a} \ll \tilde{a}_{\max }=\sqrt{1-\left(\frac{Q}{M}\right)^{2}}<1 .
$$

This condition is obviously stronger than the condition one may expect to apply a priori, i.e. $\tilde{a} \ll 1$, and it implies that the slow-rotation approximation will break down even for small values of the spin as $Q \rightarrow M$. A posteriori, this result is not too surprising, and it is consistent with the requirement that the perturbation parameter $\tilde{a}$ must be smaller than any dimensionless quantity characterizing the background spacetime.

According to Ref. [1], the slow-rotation approximation is accurate within one percent as long as $J / J_{\max } \lesssim 0.3$, and it is still accurate within $3 \%$ for $J / J_{\max } \lesssim 0.5$.

\section{B. Gravito-Electromagnetic Quasinormal Modes at First Order in Rotation}

We have computed the fundamental mode and the first overtone of the gravito-electromagnetic QNMs of a KN $\mathrm{BH}$ in the axial and polar sectors for $\ell=2$ and $\ell=3$. The numerical solution of both the axial and polar perturbation equations (2) is extremely challenging, because their explicit form is lengthy and complicated.

We have computed the axial modes using both the matrix continued-fraction method and direct integration. The results agree extremely well, so that the two methods validate each other. In the polar case the equations are too complicated to be cast in a tractable continuedfraction form, even using algebraic manipulation software like Mathematica. In this case the only viable technique turned out to be direct integration. Even the task of numerical integration is challenging due to the complexity of the field equations, but reasonably accurate results can be obtained by using high-order series expansions at the horizon and at infinity and by requiring better numerical precision in the integration. The difficulties we met in integrating the first-order equations suggest that a second-order analysis, while possible in principle, may be very challenging from the algebraic and computational standpoints. 
For any value of $Q$, our analysis allows us to extract the first-order corrections to the QNM spectrum, as defined in Eqs. (4) and (5). At first order the $m$-dependence can be factored out, so the calculation is complete once we know the functions $\omega_{R}^{(1)}$ and $\omega_{I}^{(1)}$. Furthermore, we remark that first-order corrections vanish identically when $m=0$.

We find four families of modes, two of which are associated to axial perturbations, while the other two are associated to polar perturbations. In the $Q=0$ limit the two families with a given parity reduce to the gravitational and electromagnetic modes of a Kerr BH. Therefore, with a slight abuse of notation, we will dub the two families "gravitational" and "electromagnetic" also in the general case. It should be stressed that, when $Q \neq 0$, oscillations involving any of these modes excite both electromagnetic and gravitational perturbations.

We also mention that we have carried out a further check of our results. We have extracted $\mathcal{O}(\tilde{a})$ corrections of the QNMs from a fit of the "full" numerical solution of the Teukolsky equations in the Kerr background, and we have verified that this procedure matches our results for $Q=0$ within a relative error $\lesssim 0.1 \%$ for $\omega_{R}^{(1)}(Q=0)$. The error is only a few times larger for $\omega_{I}^{(1)}(Q=0)$.

\section{Isospectrality}

For generic values of $\ell$, gravito-electromagnetic perturbations of nonspinning BHs in general relativity are isospectral [18, 44]. The left panels of Fig. 1 1 are fully consistent with isospectrality within our numerical accuracy, and this is a nontrivial consistency check of our method 1 .

A priori, there is no reason to expect that such a remarkable property should hold true also for KN BHs. Isospectrality is easily broken: for example it is well known that polar and axial modes are not isospectral (even for nonrotating $\mathrm{BHs}$ ) if the cosmological constant is nonzero 64 66], if the underlying theory is not general relativity [67, 68], or in higher dimensions [18].

Our numerics provide strong evidence that the gravitoelectromagnetic modes of $\mathrm{KN} \mathrm{BHs}$ are isospectral to first order in the angular momentum. This was shown in Fig. [ 1 for the fundamental mode with $\ell=2$ and $\ell=3$. In the insets of Fig. 1 we show the relative difference between the coefficients of axial and polar modes as functions of $Q$ : our results are consistent with isospectrality within the numerical accuracy of the direct integration method.

We believe that deviations from isospectrality are of a purely numerical nature, being almost entirely due to

\footnotetext{
1 To the best of our knowledge, in the published literature there are no studies checking isospectrality for RN BHs by an explicit calculation of polar QNMs. This is an interesting by-product of our analysis.
}

the intrinsic errors of the direct integration to compute QNMs. This issue was discussed in detail in Ref. [1]. Here we simply add the observation that, as shown in Fig. 1. the fundamental mode with $\ell=3$ (for which the direct integration is more accurate) is numerically closer to isospectrality than the fundamental mode with $\ell=$ 2. We have checked that deviations from isospectrality decrease as $\ell$ grows.

\section{Fitting formulae at first order in rotation}

Polar and axial modes are the same to first order within our numerical accuracy, but the equations for axial modes, which are listed explicitly in Eq. (2) and Appendix A1 of this paper, are much simpler. We carried out a more extensive QNM calculation working in the axial case, where our results can be verified using two independent methods. Due to isospectrality, these results cover the whole QNM spectrum of slowly rotating KN BHs.

We found that the zeroth- and first-order terms in Eqs. (4) and (5) shown in Fig. 11 are well fitted by functions of the form

$$
M \omega_{R, I}^{(0,1)}=f_{0}+f_{1} y+f_{2} y^{2}+f_{3} y^{3}+f_{4} y^{4},
$$

where we have defined a parameter $y=1-\tilde{a}_{\max }=$ $1-\sqrt{1-Q^{2} / M^{2}}$ which is in one-to-one correspondence with $Q$ (such that $y \in[0,1]$ as $Q \in[0, M]$ ), but is better suited for fitting. As discussed in Section IVA $\tilde{a}_{\max }$ seems to be the most appropriate dimensionless quantity to normalize our perturbative parameter; in some sense, the parameter $y$ measures the "distance from extremality" of the KN metric. The $\ell$ - and $n$-dependent fitting coefficients $f_{i}$ of the functions $\omega_{R}^{(0,1)}$ and $\omega_{I}^{(0,1)}$ for a selected subset of gravitational and electromagnetic modes are listed in Table \ which extends a similar Table in [1].

Our QNM calculations in the slow-rotation approximation can be seen as an empirical confirmation of the stability of the KN metric. We have looked for unstable modes in the region $0<Q<M, J \ll J_{\max }$ and for $\ell=2,3,4$ and we found none. This confirms early arguments by Mashhoon, who used calculations in the eikonal limit to make a case for the stability of the $\mathrm{KN}$ metric [45]. Notice however that Mashhoon's results apply only to perturbation modes with $\ell \gg 1$ and they rely on a geodesic analogy, rather than on a self-consistent treatment of the perturbation equations. In this sense, our findings provide the first self-consistent stability analysis of the KN metric.

\section{Comparison with the quasinormal modes of the Dudley-Finley equation}

Finally, we can compare our results against the DF equation [41, 42] to quantify the regime of validity of both 


\begin{tabular}{lc|ccccc} 
& $(\ell, n, s)$ & $f_{0}$ & $f_{1}$ & $f_{2}$ & $f_{3}$ & $f_{4}$ \\
\hline$\omega_{R}^{(0)}$ & $(2,0,1)$ & 0.4576 & 0.2659 & 0.0118 & 0.1228 & -0.1382 \\
$\omega_{R}^{(1)}$ & $(2,0,1)$ & 0.0712 & 0.0769 & 0.0596 & 0.0727 & -0.0216 \\
$\omega_{I}^{(0)}$ & $(2,0,1)$ & -0.0950 & -0.0184 & 0.0137 & 0.0132 & 0.0107 \\
$\omega_{I}^{(1)}$ & $(2,0,1)$ & 0.0007 & 0.0043 & 0.0060 & -0.0089 & 0.0366 \\
\hline$\omega_{R}^{(0)}$ & $(2,0,2)$ & 0.3737 & 0.0525 & 0.0607 & -0.0463 & -0.0070 \\
$\omega_{R}^{(1)}$ & $(2,0,2)$ & 0.0628 & 0.0676 & 0.0209 & 0.0823 & -0.0810 \\
$\omega_{I}^{(0)}$ & $(2,0,2)$ & -0.0890 & -0.0055 & 0.0024 & 0.0214 & -0.0084 \\
$\omega_{I}^{(1)}$ & $(2,0,2)$ & 0.0010 & 0.0014 & 0.0091 & 0.0174 & 0.0145 \\
\hline \hline$\omega_{R}^{(0)}$ & $(2,1,1)$ & 0.4365 & 0.2793 & 0.0125 & 0.1399 & -0.1637 \\
$\omega_{R}^{(1)}$ & $(2,1,1)$ & 0.0780 & 0.0785 & 0.0588 & 0.0776 & -0.0277 \\
$\omega_{I}^{(0)}$ & $(2,1,1)$ & -0.2907 & -0.0515 & 0.0438 & 0.0364 & 0.0363 \\
$\omega_{I}^{(1)}$ & $(2,1,1)$ & 0.0043 & 0.0138 & 0.0164 & -0.0230 & 0.1062 \\
\hline$\omega_{R}^{(0)}$ & $(2,1,2)$ & 0.3467 & 0.0546 & 0.0709 & -0.0292 & -0.0433 \\
$\omega_{R}^{(1)}$ & $(2,1,2)$ & 0.0717 & 0.0764 & 0.0020 & 0.1959 & -0.2213 \\
$\omega_{I}^{(0)}$ & $(2,1,2)$ & -0.2739 & -0.0157 & 0.0099 & 0.0668 & -0.0239 \\
$\omega_{I}^{(1)}$ & $(2,1,2)$ & 0.0065 & 0.0070 & 0.0360 & 0.0254 & 0.0905 \\
\hline \hline$\omega_{R}^{(0)}$ & $(3,0,1)$ & 0.6569 & 0.3684 & -0.0820 & 0.2851 & -0.2574 \\
$\omega_{R}^{(1)}$ & $(3,0,1)$ & 0.0726 & 0.0768 & 0.0595 & 0.0617 & -0.0259 \\
$\omega_{I}^{(0)}$ & $(3,0,1)$ & -0.0956 & -0.0177 & 0.0178 & 0.0074 & 0.0106 \\
$\omega_{I}^{(1)}$ & $(3,0,1)$ & 0.0002 & 0.0032 & 0.0027 & -0.0002 & 0.0216 \\
\hline$\omega_{R}^{(0)}$ & $(3,0,2)$ & 0.5994 & 0.0790 & 0.1734 & -0.2019 & 0.0700 \\
$\omega_{R}^{(1)}$ & $(3,0,2)$ & 0.0673 & 0.0693 & 0.0211 & 0.0791 & -0.0677 \\
$\omega_{I}^{(0)}$ & $(3,0,2)$ & -0.0927 & -0.0043 & -0.0013 & 0.0292 & -0.0130 \\
$\omega_{I}^{(1)}$ & $(3,0,2)$ & 0.0006 & 0.0014 & 0.0084 & 0.0058 & 0.0122 \\
\hline \hline$\omega_{R}^{(0)}$ & $(3,1,1)$ & 0.6418 & 0.3782 & -0.0863 & 0.3104 & -0.2875 \\
$\omega_{R}^{(1)}$ & $(3,1,1)$ & 0.0760 & 0.0786 & 0.0511 & 0.0924 & -0.0576 \\
$\omega_{I}^{(0)}$ & $(3,1,1)$ & -0.2897 & -0.0509 & 0.0508 & 0.0333 & 0.0222 \\
$\omega_{I}^{(1)}$ & $(3,1,1)$ & 0.0015 & 0.0098 & 0.0096 & -0.0050 & 0.0693 \\
\hline$\omega_{R}^{(0)}$ & $(3,1,2)$ & 0.5826 & 0.0819 & 0.1752 & -0.1753 & 0.0304 \\
$\omega_{R}^{(1)}$ & $(3,1,2)$ & 0.0713 & 0.0736 & 0.0108 & 0.1287 & -0.1282 \\
$\omega_{I}^{(0)}$ & $(3,1,2)$ & -0.2813 & -0.0123 & -0.0050 & 0.0972 & -0.0472 \\
$\omega_{I}^{(1)}$ & $(3,1,2)$ & 0.0029 & 0.0059 & 0.0194 & 0.0374 & 0.0231 \\
\hline \hline & & & & & & \\
\hline & & & & & & \\
& & & & & \\
& & & & \\
\end{tabular}

TABLE I. Coefficients of the fit (35) for the real and imaginary part of a selected subset of gravito-electromagnetic modes. The values $(\ell, n)$ correspond to the multipolar index and the overtone number, respectively. Fundamental modes correspond to $n=0$. We denote by $s=1$ and $s=2$ the modes that in the decoupled $Q \rightarrow 0$ limit are electromagnetic and gravitational in the Kerr background, respectively. The fits (35) reproduce the data to within $1 \%$ for $\omega_{I}^{(1)}$ and to within $0.1 \%$ for the other quantities for any $Q \lesssim 0.95 M$.

approximation schemes. The results of this comparison for the fundamental gravitational mode with $\ell=m=2$ are shown in Fig. 2 .

The DF equation reduces to the Teukolsky equation for Kerr BHs in the limit $Q \rightarrow 0$. However, it does not reduce to the RN Regge-Wheeler/Zerilli equations when $\tilde{a} \rightarrow 0$ [20]. On the other hand, our slow-rotation approximation is valid for any $Q$, but we must impose the condition $J \ll J_{\max }$. In the region $J \ll J_{\max }$, the slow-

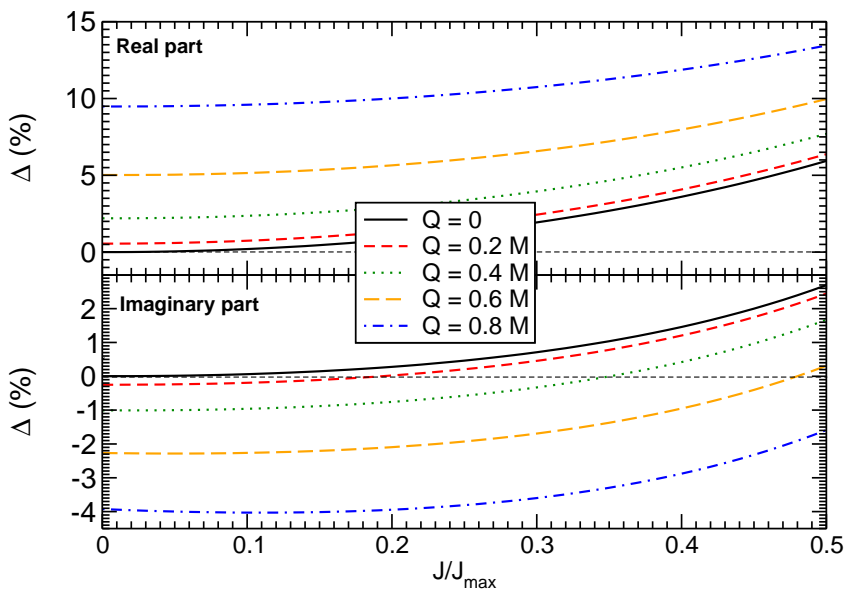

FIG. 2. Percentage deviation of QNM frequencies in the slowrotation approximation with respect to the DF equation, for the $\ell=m=2$ fundamental gravitational mode. As expected, the two approximations agree with each other when $Q \rightarrow 0$, but they deviate from each other when $\tilde{a} \rightarrow 0$. The discrepancy between the two approximations is nearly constant as long as $J \ll J_{\max }$, confirming that the DF equation is not very accurate in that regime when $Q \neq 0$ (cf. [20]).

rotation approximation can be used to quantify the errors introduced by the DF equation for any value of $Q$.

Figure 2 shows that the deviations between the DF and slow-rotation calculations vanish when $Q \ll M$ and $J \ll J_{\max }$, i.e. in the region where the assumptions underlying both approximations are consistent. As we increase $Q$ we observe an increasing deviation of the DF modes with respect to the slow-rotation calculation. The offset increases with $Q$, and it is nearly constant for any $Q$ in the region $J \ll J_{\max }$ : the errors introduced by the DF approximation in the nonrotating case do not increase much when (a small amount of) rotation is included. The deviations do increase for larger values of $J$, but in that regime the slow-rotation approximation is not reliable anymore.

\section{CONCLUSIONS}

In this paper and the accompanying Letter [1] we have presented the first fully-consistent analysis of the gravitoelectromagnetic QNMs of the KN metric. Working in a slow-rotation approximation, the long-standing problem of nonseparability of the perturbation equations can be evaded. We have computed the gravito-electromagnetic QNMs to first order in the $\mathrm{BH}$ spin and provided fitting formulae for the fundamental mode and first overtone with $\ell=2,3$.

Furthermore, our numerical study of gravitoelectromagnetic perturbations shows strong numerical evidence for the isospectrality of polar and axial gravitoelectromagnetic perturbations of KN black holes at 
linear order in rotation. It would be interesting to understand whether isospectrality holds exactly, at all orders in rotation. An important extension of our work in this direction is to include second-order effects. The causal structure of a spinning metric starts differing from the nonspinning case at second order in the angular momentum (e.g. changes in the horizon location and in the ergoregion are of second order), thus if isospectrality holds true also at second order, there is no fundamental reason to believe that it is broken at higher order.

If isospectrality is an exact property of $\mathrm{KN} \mathrm{BHs}$, extensions of the polar and axial field equations (2) to any order in the spin should be related to each other by some transformation that leaves the QNM spectrum invariant. Even at linear order in rotation isospectrality is a highly nontrivial property, in view of the mixing of gravitational and electromagnetic perturbations. Hopefully our work will stimulate further study to prove (or disprove) the conjecture that isospectrality is an exact property of the KN spacetime. This conjecture may be verified using a brute-force extension of our work to higher orders in rotation, numerical time evolutions along the lines of 69 71], or (ideally) an analytical proof, perhaps similar to Chandrasekhar's proof in the nonrotating case (see also Ref. [2]).

In Ref. 1] we have presented some possible extensions of our work. If isospectrality turns out to be valid for any value of the angular momentum, an interesting avenue of research is to understand whether such property has implications in the context of the KN/CFT conjecture [24, 25], which predicts that the QNMs of the nearhorizon KN geometry correspond to the poles of the retarded Green's function of the dual chiral CFT [73].

Some further interesting applications concern nonasymptotically flat spacetimes. Even for nonrotating RN (anti-)de Sitter BHs isospectrality is known to be partially broken, depending on the relative size of the $\mathrm{BH}$ and (anti-)de Sitter horizon radii [64 66, 74. The slow-rotation approximation may be used to understand whether similar considerations also apply to Kerr-Newman (anti-)de Sitter. Indeed, our approach can be easily extended to include a nonvanishing cosmological constant. In the context of the AdS/CFT correspondence [75], the QNMs of a $\mathrm{KN} \mathrm{BH}$ are dual to thermal states of a CFT living in a rotating Einstein universe [76, 77].

Finally, certain KN-AdS BHs embedded in $N=2$ fourdimensional supergravity preserve half of the supersymmetry [8, 79]. This is analogous to the case of asymptotically flat, extremal RN BHs, for which supersymmetry implies a remarkable property of the QNMs: electromagnetic perturbations with multipolar index $\ell$ are isospectral with gravitational perturbations with index $\ell+1$ [80]. Using this property, it is possible to prove that the one-loop corrections to the $\mathrm{BH}$ entropy cancel [81]. It would be interesting to understand whether such supersymmetry implies a similar property for the supersymmetric KN-AdS BH solutions found in Refs. 78, 79].
We note that such solutions can be slowly rotating, so our framework can be directly applied to this interesting problem.

We hope that our paper will stimulate further work in these, and possibly other, directions.

\section{ACKNOWLEDGMENTS}

We wish to thank Vitor Cardoso for useful discussions. This work was supported by the NRHEP295189 FP7-PEOPLE-2011-IRSES Grant, and by FCT - Portugal through PTDC projects FIS/098025/2008, FIS/098032/2008, CERN/FP/123593/2011. E.B. was supported by NSF CAREER Grant No. PHY-1055103. P.P. acknowledges financial support provided by the European Community through the Intra-European Marie Curie contract aStronGR-2011-298297. Computations were performed on the "Baltasar Sete-Sois" cluster at IST, the cane cluster in Poland through PRACE DECI-7 "Black hole dynamics in metric theories of gravity", on Altamira in Cantabria through BSC grant AECT-2012-30012, on Caesaraugusta in Zaragoza through BSC grants AECT-2012-2-0014 and AECT-2012-3-0011, XSEDE clusters SDSC Trestles and NICS Kraken through NSF Grant No. PHY-090003, Finis Terrae through Grant CESGA-ICTS-234.

\section{Appendix A: Derivation of the perturbation equations}

In this Appendix we derive the first-order equations (2) for the axial and polar gravito-electromagnetic perturbations of a $\mathrm{KN} \mathrm{BH}$. Various intermediate steps are presented in a supplementary MATHEMATICA notebook [56].

\section{Axial sector}

As discussed in the main text and in Ref. [51], the coupling between perturbations with different parity and different harmonic index $\ell$ does not contribute to the QNM to first order in $\tilde{a}$. Neglecting the couplings to $\ell \pm 1$ terms, the axial sector is fully described by four equations:

$$
\begin{aligned}
& 0=\Lambda \beta_{\ell}^{(L)}+i m\left[(\ell-1)(\ell+2) \chi_{\ell}^{(L)}+\tilde{\alpha}_{\ell}^{(L)}+\eta_{\ell}^{(L)}\right] \\
& 0=\Lambda t_{\ell}+i m g_{\ell},
\end{aligned}
$$

where $L=0,1,2$. The choice $L=2$ refers to one of the Maxwell equations, and $\left\{\beta_{\ell}^{(L)}, \chi_{\ell}^{(L)}, \tilde{\alpha}_{\ell}^{(L)}, \eta_{\ell}^{(L)}, t_{\ell}, g_{\ell}\right\}$ are combinations of the perturbation functions $\left\{h_{0,1}^{\ell}, u_{(4)}^{\ell}\right\}$, whose explicit form can be found online [56]. Actually, Einstein's equations imply Maxwell's equations, and only three out of the four equations above are independent. This can be easily verified 
as a consistency check of our approach. The three independent equations can be solved for the functions $h_{0}^{\ell}, h_{1}^{\ell}$ and $u_{(4)}^{\ell}$. We define the Regge-Wheeler function $\Psi^{\ell}$ as

$$
\Psi^{\ell}=\frac{F h_{1}^{\ell}}{r} .
$$

Then, from Eq. (A1) with $L=1$, we get

$h_{0}^{\ell^{\prime}}=\frac{2 F\left(\Lambda r \omega h_{0}^{\ell}-2 Q \omega u_{(4)}^{\ell}\right)+i \Lambda r\left(F(\Lambda-2)-r^{2} \omega^{2}\right) \Psi^{\ell}}{F \Lambda r^{2} \omega}$

$-\frac{i m \tilde{a} M^{2}}{F \Lambda^{2} M r^{4} \omega^{2}}\left[12 i F \Lambda M \omega h_{0}^{\ell}+4 i F^{2} \Lambda Q \omega u_{(4)}^{\ell}\right.$

$\left.+(F-1) \Lambda^{2} r\left(F(\Lambda-2)+r^{2} \omega^{2}\right) \Psi^{\ell}+8 i F^{2} Q r \omega u_{(4)}^{\ell}{ }^{\prime}\right]$.

Replacing this equation in the remaining Eqs. (A1) with $L=0$ and $L=2$ we get a system for $u_{(4)}^{\ell}$ and $\Psi^{\ell}$ only. At first order in $\tilde{a}$, the system contains third derivatives of $u_{(4)}^{\ell}$. Within our perturbative scheme, the latter can be eliminated by using the zeroth order perturbation equations. The result is a system of coupled, second-order equations for $u_{(4)}^{\ell}$ and $\Psi^{\ell}$. In order to decouple the system at zeroth order, we define a linear combination of two new functions $Z_{i}^{(-)}(i=1,2)$, such that

$$
\begin{aligned}
u_{4}^{\ell} & =\alpha_{11} Z_{1}^{(-)}+\alpha_{12} Z_{2}^{(-)}, \\
\Psi^{\ell} & =\alpha_{21} Z_{1}^{(-)}+\alpha_{22} Z_{2}^{(-)},
\end{aligned}
$$

where $\alpha_{21}=4 i \alpha_{11} \omega Q /\left(\Lambda q_{1}\right), \alpha_{22}=4 i \alpha_{12} \omega Q /\left(\Lambda q_{2}\right)$, and we have defined

$$
q_{1,2}=3 M \pm \sqrt{9 M^{2}+4(\Lambda-2) Q^{2}},
$$

such that $q_{1} q_{2}=-4 Q^{2}(\Lambda-2)$ and $q_{1}+q_{2}=6 M$. The constants $\alpha_{11}$ and $\alpha_{12}$ can be set equal to unity without loss of generality.

Replacing the linear combinations above into the equations for $u_{(4)}^{\ell}$ and $\Psi^{\ell}$ and solving for $Z_{1}^{(-)}$and $Z_{2}^{(-)}$, we obtain Eq. (2) with a "minus" superscript.

The potentials appearing in the axial sector of the perturbation equations (2) read

$$
\begin{aligned}
V_{0}^{(i,-)}= & \frac{F}{r^{3}}\left[-q_{j}+\frac{4 Q^{2}}{r}\right] \\
V_{1}^{(i,-)}= & -\frac{M}{\Lambda\left(q_{i}-q_{j}\right) r^{11} \omega}\left[4 Q^{6}\left(10 q_{i} q_{j}-12 M r+\left(-18 q_{j}+\Lambda\left(q_{i}+8 q_{j}\right)\right) r+\lambda r^{2}\right)\right. \\
& -2 M q_{j} r^{3}(r-2 M)\left(r\left((18+\Lambda) q_{i}+12(\Lambda-3) r\right)-6 M\left(7 q_{i}+(-14+5 \Lambda) r\right)\right) \\
& +r^{7}\left(2 M q_{i} q_{j}-4 \Lambda M\left(q_{i}-q_{j}\right) r-q_{i} q_{j} r\right) \omega^{2}+Q^{4} r\left(192 M^{2} r-4 M\left(50 q_{i} q_{j}+\left(-92 q_{j}+\Lambda\left(4 q_{i}+39 q_{j}\right)\right) r+4(6+\lambda) r^{2}\right)\right. \\
& \left.+r\left((72+\Lambda) q_{i} q_{j}+8 \Lambda q_{i} r+4 r\left(2(-16+7 \Lambda) q_{j}+\lambda r+(6+\Lambda) r^{3} \omega^{2}\right)\right)\right) \\
& +Q^{2} r^{2}\left(-192 M^{3} r+4 M^{2}\left(81 q_{i} q_{j}+\left(-154 q_{j}+\Lambda\left(4 q_{i}+61 q_{j}\right)\right) r+4(12+\lambda) r^{2}\right)\right. \\
& +r^{2}\left(8(3 \Lambda-7) q_{j} r+2 r^{3}\left(-\Lambda q_{j}+2(6+\Lambda) r\right) \omega^{2}+q_{i}\left(q_{j}\left(32+\Lambda-r^{2} \omega^{2}\right)+2 \Lambda r\left(2+r^{2} \omega^{2}\right)\right)\right) \\
& \left.\left.-4 M r\left((55+\Lambda) q_{i} q_{j}+4 \Lambda q_{i} r+r\left((-102+41 \Lambda) q_{j}+2 r\left(6+6 r^{2} \omega^{2}+\Lambda\left(-2+\Lambda+r^{2} \omega^{2}\right)\right)\right)\right)\right)\right] \\
V_{2}^{(i,-)}= & \frac{2 M F^{2}}{\Lambda\left(q_{i}-q_{j}\right) r^{6} \omega}\left(Q^{2}\left(q_{i}\left(5 q_{j}-4 r\right)+4\left(6 M+(\Lambda-1) q_{j}\right) r\right)+q_{j} r\left(3 q_{i} r-2 M\left(4 q_{i}+3 \Lambda r\right)\right)\right), \\
W_{1}^{(i,-)}= & \frac{4(\Lambda-2) M F}{\Lambda\left(q_{i}-q_{j}\right) q_{j}^{2} r^{9} \omega}\left[4 Q^{4}\left(10 q_{j}^{2}+9(\Lambda-2) q_{j} r+r(\lambda r-12 M)\right)+q_{j} r^{2}\left(2 M \left(r\left(-(18+\Lambda) q_{j}-12(\Lambda-3) r\right)\right.\right.\right. \\
& \left.\left.+6 M\left(7 q_{j}+(-14+5 \Lambda) r\right)\right)-q_{j} r^{4} \omega^{2}\right)+Q^{2} r\left(96 M^{2} r+q_{j} r\left((32+\Lambda) q_{j}+28(\Lambda-2) r\right)\right. \\
& \left.\left.+4 M\left(-30 q_{j}^{2}+(56-25 \Lambda) q_{j} r-2(6+\lambda) r^{2}\right)+4(6+\Lambda) r^{5} \omega^{2}\right)\right] \\
W_{2}^{(i,-)}= & \frac{8(\Lambda-2) M F^{2}}{\Lambda\left(q_{i}-q_{j}\right) q_{j}^{2} r^{6} \omega}\left(q_{j} r\left(8 M q_{j}+6 \Lambda M r-3 q_{j} r\right)+Q^{2}\left(-5 q_{j}^{2}-4\left(6 M+(\Lambda-2) q_{j}\right) r\right)\right)
\end{aligned}
$$

where $q_{i, j}(i, j=1,2$ and $i \neq j)$ are defined as in Eq. (A6), and $\lambda \equiv \Lambda(\ell+2)(\ell-1)$.

\section{Polar sector}

The equations governing the polar sector are more involved. They can obtained by following Zerilli's original derivation of the gravitational and electromagnetic perturbations of a RN BH [59], extended to first order in the $\mathrm{BH}$ spin.
Polar perturbations are fully described by the equa- 
tions:

$$
\begin{aligned}
& 0=A_{\ell}^{(I)}+i m C_{\ell}^{(I)}, \\
& 0=\Lambda \alpha_{\ell}^{(L)}+i m\left[(\ell-1)(\ell+2) \xi_{\ell}^{(L)}-\tilde{\beta}_{\ell}^{(L)}-\zeta_{\ell}^{(L)}\right] \\
& 0=\Lambda s_{\ell}-i m f_{\ell},
\end{aligned}
$$

where $I=0, \ldots, 5$ and $L=0,1,2$, and $\left\{A_{\ell}^{(I)}, C_{\ell}^{(I)}, \alpha_{\ell}^{(L)}, \xi_{\ell}^{(L)}, \tilde{\beta}_{\ell}^{(L)}, \zeta_{\ell}^{(L)}, s_{\ell}, f_{\ell}\right\}$ are combinations of the perturbation functions $\left\{H_{0,1,2}^{\ell}, K^{\ell}, u_{(1,2,3)}^{\ell}\right\}$, whose explicit form can be found online [56]. Actually, only seven out of the ten equations above are independent and they can be solved for the seven polar functions: $H_{0}^{\ell}, H_{1}^{\ell}, H_{2}^{\ell}, K^{\ell}, u_{(i)}^{\ell}(i=1,2,3)$.

The key point of Zerilli's calculation is to use perturbations of the field strenght $F_{\mu \nu}$, rather than the electromagnetic potential $A_{\mu}$, as dynamical variables. Following [59], we define

$$
\delta F_{\mu \nu} \equiv f_{\mu \nu}=\partial_{\mu} \delta A_{\nu}-\partial_{\nu} \delta A_{\mu} .
$$

In the polar sector, we fix the gauge by requiring $u_{(3)}^{\ell}=$ 0 ; the remaining components are related to $f_{\mu \nu}$ in the following way

$$
\begin{aligned}
u_{(1)}^{\ell} & =r \tilde{f}_{02}, \\
u_{(2)}^{\ell} & =F(r) \tilde{f}_{12}, \\
u_{(1)}^{\ell}{ }^{\prime} & =r \tilde{f}_{01}+\tilde{f}_{02}-i r \omega \tilde{f}_{02},
\end{aligned}
$$

where $-\tilde{f}_{\mu \nu}$ denotes the angle-independent part of $f_{\mu \nu}$ (note that our definition differs from Zerilli's definition by a minus sign). The equation

$$
\tilde{f}_{01}=\tilde{f}_{02}^{\prime}+i \omega \tilde{f}_{12}
$$

is automatically satisfied due to Eq. A15.

First, we solve Eq. A14 for $H_{2}^{\ell}$ and substitute the result into the remaining equations. Then, Eq. A12 with $I=5$ and Eq. A13 with $L=2$ can be solved for $\tilde{f}_{01}$ and $\tilde{f}_{02}$. The solutions can be replaced into Eq. (A19), which takes the form of a second-order differential equation for $\tilde{f}_{12}$ with source terms linear in the polar gravitational function $H_{0}^{\ell}, H_{1}^{\ell}, K^{\ell}$ and in their first derivatives.

The equations for the gravitational sector can be obtained by solving Eq. A12 with $I=1$ and Eqs. A13 with $L=0,1$ for $H_{0}^{\ell^{\prime}}, H_{1}^{\ell^{\prime}}$ and $K^{\ell^{\prime}}$. Similarly to the axial sector, second derivatives of these perturbation functions (which appear at first order in $\tilde{a}$ ) can be eliminated using the zeroth-order equations. By substituing the solution into Eq. A12 with $I=2$, one can solve for the function $H_{0}^{\ell}$ and eliminate it from the remaining equations. As a result of this procedure we obtain a system of coupled equations

$$
\mathbf{Y}^{\prime}+\mathbf{U Y}=0
$$

where $\mathbf{Y}=\left(H_{1}^{\ell}, K^{\ell}, \tilde{f}_{12}, \tilde{f}_{12}^{\prime}\right)$ and $\mathbf{U}$ is a matrix. The system above collectively denotes two first-order equations for the gravitational perturbations $H_{0}^{\ell}$ and $K^{\ell}$ and the second-order equation for $\tilde{f}_{12}$, which has been separated into two first order equations for $\tilde{f}_{12}$ and $\tilde{f}_{12}^{\prime}$.

As shown by Zerilli [59], at zeroth order in the BH spin the two equations for the gravitational perturbations $H_{0}^{\ell}$ and $K^{\ell}$ can be reduced to a single, second-order equation. We describe the procedure here, extending it to first order in the $\mathrm{BH}$ spin. The perturbations $H_{0}^{\ell}$ and $K^{\ell}$ satisfy the schematic matrix-valued equation

$$
\mathbf{y}^{\prime}=\mathbf{A y}+\mathbf{S}
$$

where $\mathbf{A}$ is a matrix, $\mathbf{y}=\left(K^{\ell}, H_{1}^{\ell} / \omega\right)$ and $\mathbf{S}=\left(S_{1}, S_{2}\right)$ is a source term, which depends on $\tilde{f}_{12}$ and its derivatives only. The idea is to find a transformation $\mathbf{y}=\mathbf{F} \hat{\mathbf{y}}$ to a new pair of functions $\hat{\mathbf{y}}=\left(\hat{\Psi}_{1}, \hat{\Psi}_{2}\right)$ such that

$$
\begin{aligned}
& \frac{d \hat{\Psi}_{1}}{d \hat{r}}=G_{1} \hat{\Psi}_{1}+(1+W) \hat{\Psi}_{2}+\hat{S}_{1}, \\
& \frac{d \hat{\Psi}_{2}}{d \hat{r}}=-\left(\omega^{2}-V-V_{1}\right) \hat{\Psi}_{1}+G_{2} \hat{\Psi}_{2}+\hat{S}_{2},
\end{aligned}
$$

where $\hat{r}$ is a new variable defined by $d r / d \hat{r}=n(r)$. Indeed, assuming the relations above, we can solve Eq. A22 for $\hat{\Psi}_{2}$ and substitute it into Eq. A23. We obtain a single, second-order equation for $\hat{\Psi}_{1}$ :

$$
\frac{d^{2} \hat{\Psi}_{1}}{d \hat{r}^{2}}+\hat{U} \frac{d \hat{\Psi}_{1}}{d \hat{r}}+\hat{V} \hat{\Psi}_{1}=\hat{\mathcal{S}}
$$

where, to first order,

$$
\begin{aligned}
& \hat{U}=-\frac{G_{1}+G_{2}+F W^{\prime}}{F^{\prime} F}, \\
& \hat{V}=\left(\omega^{2}-V\right)(1+W)-V_{1}-F G_{1}^{\prime}, \\
& \hat{\mathcal{S}}=(1+W) \hat{S}_{2}+F \hat{S}_{1}^{\prime}-\hat{S}_{1}\left(G_{2}+F W^{\prime}\right) .
\end{aligned}
$$

Note that $G_{i}, V_{1}$ and $W$ are first-order quantities in rotation, which are absent in the nonrotating case discussed in Ref. 59].

Let us now find the explicit form of the transformation. Using Eq. A21, we get

$$
\frac{d \hat{\mathbf{y}}}{d \hat{r}}=n(r) \mathbf{F}^{-1}\left[\mathbf{A} \mathbf{F}-\frac{d \mathbf{F}}{d r}\right]+n(r) \mathbf{F}^{-1} \mathbf{S} .
$$

Therefore, the transformation matrix $\mathbf{F}$ and the remaining functions must satisfy

$$
n(r) \mathbf{F}^{-1}\left[\mathbf{A} \mathbf{F}-\frac{d \mathbf{F}}{d r}\right]=\left(\begin{array}{cc}
G_{1} & 1+W \\
-\omega^{2}+V+V_{1} & G_{2}
\end{array}\right),
$$

and $\hat{\mathbf{S}}=n \mathbf{F}^{-1} \mathbf{S}$. The equation above can be solved perturbatively. At zeroth order it provides a system of four equations that can be uniquely solved for the elements of 
the matix $\mathbf{F}$, for $n(r)$ and for the potential $V$. We get

$$
\begin{aligned}
\mathbf{F}_{11}= & {\left[2 r^{3}\left(r(6 M+(\Lambda-2) r)-4 Q^{2}\right)\right]^{-1} } \\
& \times\left[16 Q^{4}-4 Q^{2} r(11 M+(\Lambda-4) r)\right. \\
& \left.+r^{2}\left(24 M^{2}+6(\Lambda-2) M r+\lambda r^{2}\right)\right], \\
\mathbf{F}_{12}= & 1, \\
\mathbf{F}_{21}= & -i\left[1+\frac{Q^{2}-M r}{r^{2} F}+\frac{8 Q^{2}-6 M r}{r[6 M+(\Lambda-2) r]-4 Q^{2}}\right], \\
\mathbf{F}_{22}= & -\frac{i r}{F},
\end{aligned}
$$

and

$$
\begin{aligned}
V(r)= & \frac{F}{r^{2}}\left(r(6 M+(\Lambda-2) r)-4 Q^{2}\right)^{-2} \\
& \times\left[-32 Q^{6}+24 Q^{4} r(6 M+(\Lambda-2) r)\right. \\
& +8 Q^{2} r^{2}\left(3(\Lambda-2) r^{2}-27 M^{2}-2(4 \Lambda-11) M r\right) \\
& +r^{3}\left(72 M^{3}+36(\Lambda-2) M^{2} r+6(\Lambda-2)^{2} M r^{2}\right. \\
& \left.\left.+\Lambda(\Lambda-2)^{2} r^{3}\right)\right], \\
n(r)= & F(r) .
\end{aligned}
$$

Note that, by virtue of the field equations, the coordinate $\hat{r}$ is the standard tortoise coordinate. The equations above correct some typos in Ref. [59]. At first order, we can solve the four equations A29 for the variables $G_{1}$, $G_{2}, W$ and $V_{1}$. The lengthy form of these solutions is presented online [56].

With the transformation at hand, we can compute the explicit form of Eq. (A24). The source term $\hat{\mathcal{S}}$ explicitly depends on the second derivatives of $\tilde{f}_{12}$ and, upon substitution of the perturbation equation for $\tilde{f}_{12}$, it depends on $K^{\ell}$ and $H_{1}^{\ell}$. This dependence can be eliminated using the definitions

$$
\begin{aligned}
& K^{\ell}=\mathbf{F}_{11} \hat{\Psi}_{1}+\frac{\mathbf{F}_{12}}{1+W}\left[F \hat{\Psi}_{1}^{\prime}-G_{1} \hat{\Psi}_{1}-\hat{S}_{1}\right], \\
& H_{1}^{\ell}=\omega \mathbf{F}_{21} \hat{\Psi}_{1}+\frac{\omega \mathbf{F}_{22}}{1+W}\left[F \hat{\Psi}_{1}^{\prime}-G_{1} \hat{\Psi}_{1}-\hat{S}_{1}\right],
\end{aligned}
$$

which allows us to write $K^{\ell}$ and $H_{1}^{\ell}$ in terms of $\hat{\Psi}_{1}$ and its first derivative only. When inserted into Eq. (A24), the equations above introduce extra coefficients in front of $d \hat{\Psi}_{1} / d r_{*}$ and $\hat{\Psi}_{1}$. The final result is an equation of the same form as Eq. A24, with coefficients given in 56.

To summarize, we have obtained two second-order equations for the functions $\hat{\Psi}_{1}$ and $\tilde{f}_{12}$ which describe gravitational and electromagnetic perturbations, respectively. At zeroth order in rotation, these equations can be decoupled [44, 82] by introducing the functions $Z_{i}^{(+)}$ such that

$$
\begin{aligned}
\hat{\Psi}_{1} & =B_{11} Z_{1}^{(-)}+B_{12} Z_{2}^{(+)}, \\
\tilde{f}_{E M} & =B_{21} Z_{1}^{(+)}+B_{22} Z_{2}^{(+)},
\end{aligned}
$$

where $B_{i j}$ are functions of $r$ and we defined $\tilde{f}_{E M} \equiv F \tilde{f}_{12}$. It is straightforward to verify that with the choice

$$
\begin{aligned}
\frac{B_{11}}{\alpha}+q_{2} & =\frac{B_{12}}{\beta}+q_{1}=\frac{4 Q^{2}}{r}, \\
\frac{B_{21}}{\alpha} & =\frac{B_{22}}{\beta}=-\frac{8 i Q}{\omega},
\end{aligned}
$$

the final set of equations takes the form of Eq. (2) with a "plus" superscript, i.e., the equations are decoupled at zeroth order (but coupled at first order) in $\tilde{a}$. In the equations above, $\alpha$ and $\beta$ are constants that can be set to unity without loss of generality. Our equations reduce to those obtained by Chandrasekhar [44] in the nonspinning case, as they should. Let us stress again that the derivation sketched in this Appendix and in 56 corrects some typos in Ref. 59].

The potentials appearing in the perturbation equations (2) of the polar sector are very lengthy [56], and their practical use may be limited. However, since - according to our numerical evidence - axial and polar modes are isospectral to linear order in $\tilde{a}$, the potentials describing axial perturbations (explicitly listed in Appendix A 1 ) are sufficient to compute the entire QNM spectrum of slowly rotating $\mathrm{KN}$ BHs.

\section{Appendix B: Coefficients of the recurrence relation for scalar QNMs of a KN BHs}

Using the same ansatz as in Eq. (29), Eq. (33) reduce to a five-term recurrence relation

$$
\begin{array}{ll}
\alpha_{0} a_{1}+\beta_{0} a_{0}=0, & n=0, \\
\alpha_{1} a_{2}+\beta_{1} a_{1}+\gamma_{1} a_{0}=0, & n=1, \\
\alpha_{2} a_{3}+\beta_{2} a_{2}+\gamma_{2} a_{1}+\delta_{2} a_{0}=0, & n=2, \\
\alpha_{n} a_{n+1}+\beta_{n} a_{n}+\gamma_{n} a_{n-1}+\delta_{n} a_{n-2}+\rho_{n} a_{n-3}=0, & n>2,
\end{array}
$$


whose coefficients to first order in $\tilde{a}$, using Leaver's $2 M=1$ unit convention, read:

$$
\begin{aligned}
\alpha_{n}= & (1+n) \Delta_{Q}\left(1+\Delta_{Q}\right)^{2}\left(2(1+n) \Delta_{Q}-i\left(1+\Delta_{Q}\right)^{2} \omega\right)+2 i m \tilde{a}(1+n) \Delta_{Q}\left(1+\Delta_{Q}\right)^{2}, \\
\beta_{n}= & \Delta_{Q}\left(1+\Delta_{Q}\right)\left(-2 \Delta_{Q}\left(-1+4 n^{2}+3 \Delta_{Q}+\ell(1+\ell)\left(1+\Delta_{Q}\right)+n\left(-2+6 \Delta_{Q}\right)\right)\right. \\
& \left.+i\left(1+\Delta_{Q}\right)^{2}\left(-1+5 \Delta_{Q}+4 n\left(1+\Delta_{Q}\right)\right) \omega+2\left(1+\Delta_{Q}\right)^{4} \omega^{2}\right) \\
& -2 m \tilde{a} \Delta_{Q}\left(1+\Delta_{Q}\right)\left(i\left(4 n+3 \Delta_{Q}-1\right)+\left(1+\Delta_{Q}\right)\left(2+\Delta_{Q}\right) \omega\right) \\
\gamma_{n}= & \Delta_{Q}\left(2 \Delta_{Q}\left(6 n\left(\Delta_{Q}-1\right)\left(3+\Delta_{Q}\right)-2 n^{2}\left(\Delta_{Q}^{2}-3\right)-\left(\Delta_{Q}-1\right)\left(11-7 \Delta_{Q}+2 \ell(1+\ell)\left(1+\Delta_{Q}\right)\right)\right)\right. \\
& \left.+i\left(1+\Delta_{Q}\right)^{2}\left(9+6 \Delta_{Q}-15 \Delta_{Q}^{2}+2 n\left(-3+\Delta_{Q}\left(-6+5 \Delta_{Q}\right)\right)\right) \omega+2\left(1+\Delta_{Q}\right)^{4}\left(-3+2 \Delta_{Q}\right) \omega^{2}\right) \\
& +2 m \tilde{a} \Delta_{Q}\left(3 i\left(\Delta_{Q}-1\right)\left(3+\Delta_{Q}\right)-2 i n\left(\Delta_{Q}^{2}-3\right)-2\left(1+\Delta_{Q}\right)\left(\Delta_{Q}^{2}-3\right) \omega\right) \\
\delta_{n}= & \left(\Delta_{Q}-1 \Delta_{Q}\left(2 \Delta_{Q}\left(29+4 n^{2}+\ell+\ell^{2}-\left(21+\ell+\ell^{2}\right) \Delta_{Q}+n\left(-22+6 \Delta_{Q}\right)\right)\right.\right. \\
& \left.+i\left(1+\Delta_{Q}\right)\left(11+\left(38-17 \Delta_{Q}\right) \Delta_{Q}+4 n\left(-1+\left(-4+\Delta_{Q}\right) \Delta_{Q}\right)\right) \omega+2\left(\Delta_{Q}-3\right)\left(1+\Delta_{Q}\right)^{3} \omega^{2}\right) \\
& +2 m \tilde{a}\left(\Delta_{Q}-1\right) \Delta_{Q}\left(-11 i+4 i n+6 \omega+\Delta_{Q}\left(3 i-\left(\Delta_{Q}-3\right) \omega\right)\right) \\
\rho_{n}= & \left(\Delta_{Q}-1\right)^{2} \Delta_{Q}(-4+n-2 i \omega)\left(2(-4+n) \Delta_{Q}-i\left(1+\Delta_{Q}\right)^{2} \omega\right)+2 i m \tilde{a}\left(\Delta_{Q}-1\right)^{2} \Delta_{Q}(-4+n-2 i \omega)
\end{aligned}
$$

where $\Delta_{Q}=\sqrt{1-4 Q^{2}}$.

[1] P. Pani, E. Berti, and L. Gualtieri, Phys.Rev.Lett. 110, 241103 (2013), arXiv:1304.1160 [gr-qc].

[2] P. T. Chrusciel, J. L. Costa, and M. Heusler, Living Rev.Rel. 15, 7 (2012), arXiv:1205.6112 gr-qc].

[3] E. T. Newman, R. Couch, K. Chinnapared, A. Exton, A. Prakash, et al., J.Math.Phys. 6, 918 (1965).

[4] B. Carter, Phys.Rev. 174, 1559 (1968).

[5] C. Pekeris and K. Frankowski, Phys.Rev. A39, 518 (1989).

[6] G. Gibbons, Commun.Math.Phys. 44, 245 (1975),

[7] R. Blandford and R. Znajek, Mon.Not.Roy.Astron.Soc. 179, 433 (1977).

[8] N. Dadhich and Z. Y. Turakulov, Class.Quant.Grav. 19, 2765 (2002), arXiv:gr-qc/0112031 [gr-qc

[9] W. Unruh, Phys. Rev. Lett. 31, 1265 (1973).

[10] S. Chandrasekhar, Proc.Roy.Soc.Lond. A349, 571 (1976).

[11] D. N. Page, Phys.Rev. D14, 1509 (1976).

[12] G. Torres del Castillo and G. Silva-Ort:goza, Phys.Rev. D42, 4082 (1990).

[13] B. Carter and R. Mclenaghan, Phys.Rev. D19, 1093 (1979).

[14] B. Carter, AIP Conf.Proc. 841, 29 (2006), arXiv:gr-qc/0604064 [gr-qc]

[15] K. D. Kokkotas and B. G. Schmidt, Living Rev.Rel. 2, 2 (1999), arXiv:gr-qc/9909058 [gr-qc].

[16] H.-P. Nollert, Class.Quant.Grav. 16, R159 (1999).

[17] V. Ferrari and L. Gualtieri, Gen.Re].Grav. 40, 945 (2008), arXiv:0709.0657 [gr-qc].

[18] E. Berti, V. Cardoso, and A. O. Starinets, Class.Quant.Grav. 26, 163001 (2009), arXiv:0905.2975 $|\mathrm{gr}-\mathrm{qc}|$.

[19] R. Konoplya and A. Zhidenko, Rev.Mcd.Phys. 83, 793 (2011), arXiv:1102.4014 [gr-qc].

[20] E. Berti and K. D. Kokkotas, Phys.Rev. D71, 124008 (2005), arXiv:gr-qc/0502065 [gr-qc]

[21] R. Konoplya and A. Zhidenko, (2013), arXiv:1307.1812 [gr-qc]

[22] J.-l. Jing and Q.-y. Pan, Nucl.Phys. B728, 109 (2005), arXiv:gr-qc/0506098 [gr-qc]

[23] H. Furuhashi and Y. Nambu, Prog.Theor.Phys. 112, 983 (2004), arXiv:gr-qc/0402037 [gr-qc],

[24] T. Hartman, W. Song, and A. Strominger, JHEP 1003, 118 (2010), arXiv:0908.3909 [hep-th].

[25] T. Hartman, K. Murata, T. Nishioka, and A. Strominger, JHEP 0904, 019 (2009), arXiv:0811.4393 [hep- th].

[26] S. Teukolsky and W. Press, Astrophys.J. 193, 443 (1974)

[27] S. Wash and N. Dadhich, Phys.Rev. D32, 1863 (1985)

[28] F. Belgiorno and M. Martellini, Phys.Lett. B453, 17 (1999), arXiv:gr-qc/9811060 [gr-qc].

[29] F. Finster, N. Kamran, J. Smoller, and S.T. Yau, Commun.Pure Appl.Math. 53, 902 (2000), arXiv:gr-qc/9905047 [gr-qc]

[30] F. Finster, N. Kamran, J. Smoller, and S.T. Yau, Adv.Theor.Math.Phys. 7, 25 (2003), arXiv:gr-qc/0005088 [gr-qc]

[31] D. Batic, H. Schmid, and M. Winklmeier, J.Math.Phys. 46, 012504 (2005), arXiv:math-ph/0402047 [math-ph].

[32] D. Batic and H. Schmid, (2005), arXiv:gr-qc/0512112 $[\mathrm{gr}-\mathrm{qc}]$,

[33] D. Batic and H. Schmid, Prog.Theor.Phys. 116, 517 (2006), arXiv:gr-qc/0606050 [gr-qc].

[34] M. Wilaklmeier and O. Yamada, J.Math.Phys. 47, 102503 (2006), arXiv:gr-qc/0605146 [gr-qc].

[35] X. He and J. Jing, Nucl.Phys. B755, 313 (2006) arXiv:gr-qc/0611003 [gr-qc]

[36] S. Zhou and W. Liu, Phys.Rev. D77, 104021 (2008)

[37] S. Dolan and J. Gair, Class.Quant.Grav. 26, 175020 (2009), arXiv:0905.2974 [gr-qc]

[38] F. Belgiorno and S. L. Cacciatori, J.Math.Phys. 51, 033517 (2010), arXiv:0803.2496 [math-ph].

[39] E. Leaver, Proc.Roy.Soc.Lond. A402, 285 (1985).

[40] H. Cho, A. Cornell, J. Doukas, T. Huang, and W. Naylor, 
Adv.Math.Phys. 2012, 281705 (2012) arXiv:1111.5024 [gr-qc].

[41] A. L. Dudley and J. Finley, Phys.Rev.Lett. 38, 1505 (1977).

[42] A. L. Dudley and I. Finley, J.D., J.Math.Phys. 20, 311 (1979).

[43] V. Bellezza and V. Ferrari, J.Math.Phys. 25, 1985 (1984).

[44] S. Chandrasekhar, The mathematical theory of black holes (1983).

[45] B. Mashhoon, Phys. Rev. D 31, 290 (1985)

[46] C. Goebel, Astrophys.J.Lett. 172, L95 (1972)

[47] V. Cardoso, A. S. Miranda, E. Berti, H. Witek, and V. T. Zanchin, Phys.Rev. D79, 064016 (2009), arXiv:0812.1806 [hep-th]

[48] J. Plebanski and M. Demianski, Annals Phys. 98, 98 (1976).

[49] K. D. Kokkotas, Nuovo Cimento 108, 991 (1993).

[50] P. Pani, V. Cardoso, L. Gualtieri, E. Berti, and A. Ishibashi, Phys.Rev.Lett. 109, 131102 (2012). arXiv:1209.0465 [gr-qc]

[51] P. Pani, V. Cardoso, L. Gualtieri, E. Berti, and A. Ishibashi, Phys.Rev. D86, 104017 (2012) arXiv:1209.0773 [gr-qc]

[52] S. Chandrasekhar and V. Ferrari, Proc.Roy.Soc.Lond. A433, 423 (1991).

[53] Y. Kojima, Phys.Rev. D46, 4289 (1992).

[54] Y. Kojima, Astrophys.J. 414, 247 (1993).

[55] V. Ferrari, L. Gualtieri, and S. Marassi, Phys.Rev. D76, 104033 (2007), arXiv:0709.2925 [gr-qc]

[56] http://blackholes.ist.utl.pt/?page=Files, http://www.phy.olemiss.edu/ berti/qnms.html

[57] P. Pani, (2013), arXiv:1305.6759 [gr-qc]

[58] E. Berti, V. Cardoso, and C. M. Will, Phys.Rev. D73, 064030 (2006), arXiv:gr-qc/0512160 [gr-qc]

[59] F. Zerilli, Phys.Rev. D9, 860 (1974)

[60] E. W. Leaver, Phys.Rev. D41, 2986 (1990)

[61] J. G. Rosa and S. R. Dolan, Phys.Rev. D85, 044043 (2012), arXiv:1110.4494 [hep-th].

[62] Y. Kojima, Progress of Theoretical Physics 90, 977 (1993).

[63] E. Berti, Conf.Proc. C0405132, 145 (2004),
arXiv:gr-qc/0411025 [gr-qc]

[64] F. Mellor and I. Moss, Phys.Rev. D41, 403 (1990).

[65] V. Cardoso and J. P. Lemos, Phys.Rev. D64, 084017 (2001), arXiv:gr-qc/0105103 [gr-qc].

[66] E. Berti and K. Kokkotas, Phys.Rev. D67, 064020 (2003), arXiv:gr-qc/0301052 [gr-qc].

[67] V. Cardoso and L. Gualtieri, Phys.Rev. D80, 064008 (2009), arXiv:0907.5008 [gr-qc]

[68] C. Molina, P. Pani, V. Cardoso, and L. Gualtieri, Phys.Rev. D81, 124021 (2010), arXiv:1004.4007 [gr-qc].

[69] E. N. Dorband, E. Berti, P. Diener, E. Schnetter, and M. Tiglio, Phys.Rev. D74, 084028 (2006), arXiv:gr-qc/0608091 [gr-qc]

[70] H. Witek, V. Cardoso, A. Ishibashi, and U. Sperhake, (2012), arXiv:1212.0551 [gr-qc]

[71] S. R. Dolan, (2012), arXiv:1212.1477 [gr-qc]

[72] P. T. Leung, A. Maassen van den Brink, W. M. Suen, C. W. Wong, and K. Young, ArXiv Mathematical Physics e-prints (1999), arXiv:math-ph/9909030.

[73] B. Chen and C.-S. Chu, JHEP 1005, 004 (2010) arXiv:1001.3208 [hep-th]

[74] B. Wang, C.-Y. Lin, and E. Abdalla, Phys.Lett. B481, 79 (2000), arXiv:hep-th/0003295 [hep-th].

[75] J. M. Maldacena, Adv.Theor.Math.Phys. 2, 231 (1998), arXiv:hep-th/9711200 [hep-th]

[76] M. M. Caldarelli, G. Cognola, and D. Klemm, Class.Quant.Grav. 17, 399 (2000), arXiv:hep-th/9908022 [hep-th].

[77] S. Hawking and H. Reall, Phys.Rev. D61, 024014 (2000) arXiv:hep-th/9908109 [hep-th]

[78] V. A. Kostelecky and M. J. Perry, Phys.Lett. B371, 191 (1996), arXiv:hep-th/9512222 [hep-th].

[79] M. M. Caldarelli and D. Klemm, Nucl.Phys. B545, 434 (1999), arXiv:hep-th/9808097 [hep-th].

[80] H. Onozawa, T. Mishima, T. Okamura, and H. Ishihara, Phys.Rev. D53, 7033 (1996), arXiv:gr-qc/9603021 [gr-qc].

[81] R. Kallosh, J. Rahmfeld, and W. K. Wong, Phys.Rev. D57, 1063 (1998), arXiv:hep-th/9706048 [hep-th]

[82] V. Moncrief, Phys.Rev. D12, 1526 (1975). 